\title{
Efficient hole transport material formed by atmospheric pressure plasma functionalization of Spiro-OMeTAD
}

Paheli Ghosh a, Aruna Ivaturi b*, Debabrata Bhattacharya c, James Bowen a, Tony Nixon a, Jan Kowal a Nicholas St. J. Braithwaite d and Satheesh Krishnamurthy a* a School of Engineering and Innovation, The Open University, Milton Keynes, MK7 6AA, United Kingdom

b Smart Materials Research and Device Technology (SMaRDT) Group, Department of Pure and Applied Chemistry, University of Strathclyde, Thomas Graham building, Glasgow, G1 1XL, United Kingdom

c School of Aerospace, Transport and Manufacturing, Cranfield University, MK43 0AL, United Kingdom

dSchool of Physical Sciences, The Open University, Milton Keynes, MK7 6AA, United Kingdom

*satheesh.krishnamurthy@open.ac.uk and aruna.ivaturi@strath.ac.uk

\begin{abstract}
A technique to increase the conductivity of Spiro-OMeTAD using an easily scalable, nonthermal atmospheric pressure plasma jet (APPJ) is reported. An investigation of plasma functionalization demonstrated an enhancement in hole conductivity by over an order of magnitude from $9.4 \times 10_{-7} \mathrm{~S} \mathrm{~cm}-1$ for the pristine film to $1.15 \times 10_{-5} \mathrm{~S} \mathrm{~cm}-1$ for films after 5 minutes of plasma treatment. The conductivity value after plasma functionalization was comparable to that reported for $10-25 \%$ Li-TFSI-doped Spiro-OMeTAD. The increase in conductivity was correlated with a reduction in phase value observed using electrostatic force microscopy. Kelvin probe force microscopy showed an increase in work function after plasma
\end{abstract}


exposure corresponding to the $p$-type nature of the doping. X-ray photoelectron spectroscopy revealed surface oxidation of plasma-functionalized films, as well as variation in nitrogen chemistry, with the formation of a higher binding energy quaternary nitrogen tail. Oxidation of Spiro-OMeTAD was also confirmed by the appearance of the $500 \mathrm{~nm}$ absorption peak using UV-vis spectroscopy. The synergistic contribution of increase in charge density in SpiroOMeTAD due to the energetic species in the plasma jet coupled with improvement in $\pi$ - $\pi$ stacking of the molecules is thought to underlie the conductivity enhancement. We also attribute the formation of quinoid structures with quaternary nitrogen $+\mathrm{N}=\mathrm{C}$ to the enhancement in positive charge centres due to loss of methoxy groups during plasma-surface interaction. This work opens up the possibility of using an atmospheric pressure plasma jet as a simple and effective technique for doping and functionalizing Spiro-OMeTAD thin films to circumvent the detrimental issues associated with chemical dopants.

\section{Graphical Abstract}

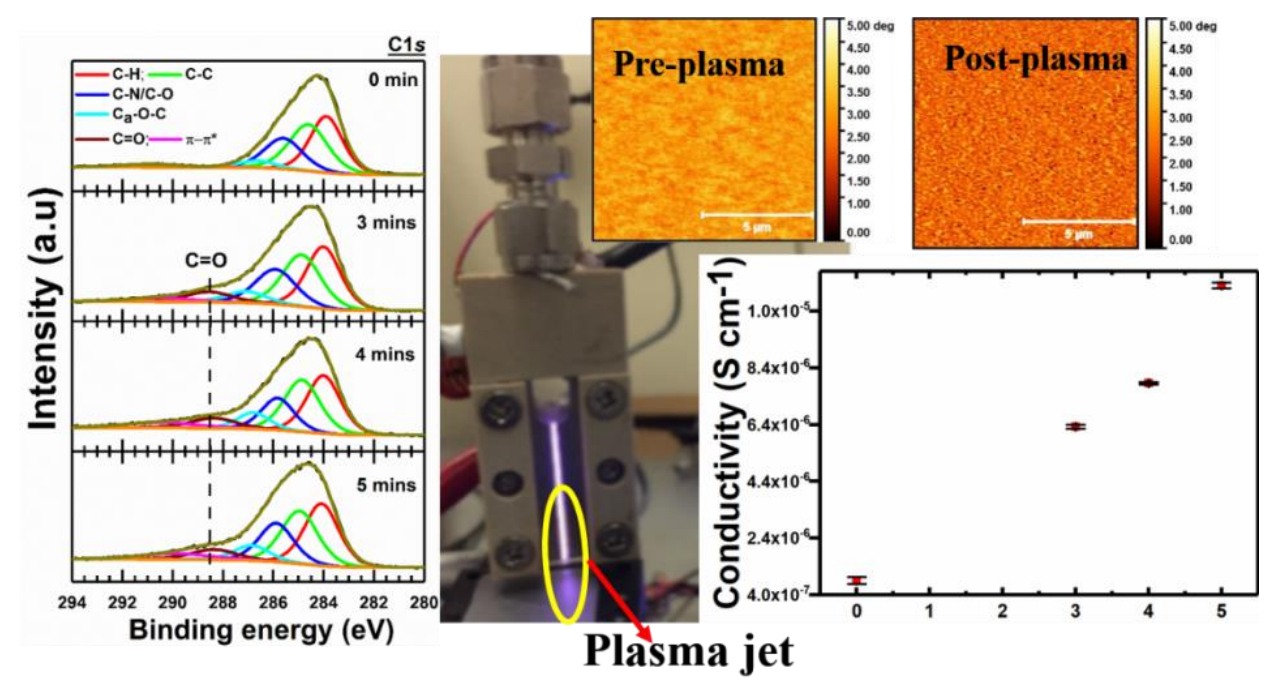

\section{KEYWORDS}

Atmospheric pressure plasma jet, conductivity, functionalization, hole transport materials, perovskite solar cells, XPS, EFM, KPFM 


\section{INTRODUCTION}

Over recent years hybrid organic-inorganic metal halide perovskite solar cells (PSCs) have achieved $>25 \%$ efficiency through judicious manipulation of perovskite morphology and precise engineering of interfacial contacts [1]. Although this efficiency is promising, one of the major obstacles to commercialisation is the stability of the active materials and several different approaches have been implemented to improve device stability [2]-[4]. A typical planar PSC consists of a perovskite absorber layer sandwiched between the electron transport layer (ETL) and the hole transport layer (HTL) with 'top' and 'bottom' electrodes formed from metallic material and transparent conducting oxide. The hole transport layer serves a two-fold purpose in defining the efficiency/performance and the stability of PSCs by (i) extraction of positive charges (holes) and subsequent transport/diffusion to the top electrode and (ii) prevention of direct contact between perovskite absorber and metal electrode, which minimizes recombination and impedes moisture ingress into the underlying perovskite absorber layer in the n-i-p architecture [5]-[7].

2,2',7,7'-Tetrakis[N,N-di-(4-methoxyphenyl)amino]9,9'-pirobifluorene], commonly known as Spiro-OMeTAD, is one of the most widely used triarylamine-based hole transport materials (HTM) for PSCs, associated with some of the best device performance to date [8]. Spiro-based small molecules, with two molecular $\pi$-systems connected by a tetrahedral carbon, i.e. the Spiro-bifluorene core, are structurally stable and the $s p 3$ hybridized carbon induces molecular rigidity, impedes crystallization below the glass transition temperature $\left(T_{g}\right)$, and enhances stability of the amorphous state, a property which is desirable in HTMs [9]. Moreover, Spiro compounds have high solubility as a result of the perpendicular arrangement of the fluorene units which suppresses the intermolecular $\pi-\pi$ impedances, resulting in solubility enhancement [10]-[12]. These favourable attributes make Spiro-OMeTAD attractive for application as HTM. However, Spiro-OMeTAD in its pristine form, has a relatively low hole mobility $(\sim 10-5 \mathrm{~cm} 2$ 
$\mathrm{V}-1 \mathrm{~S}-1)$ [10], [12], [13] and a correspondingly low intrinsic conductivity ( 10-7 $\mathrm{S} \mathrm{cm}-1)$ [13], [14]. Tuning the type and density of charge carriers via electronic doping is a suitable technique for enhancing the controllability and reproducibility of device performance [13], [15]-[19]. Doping not only affects the density of charge carriers but also tunes the electronic properties, and dopants such as Na-TFSI [20], Ag-TFSI [21], Co (III) complexes [14], [22], WO3 [23], and $\mathrm{Cu}(\mathrm{II})$ salts [24], [25] have been explored in the case of Spiro-OMeTAD [15], [25]-[27]. However, some of the $p$-type dopants exhibit poor solubility in organic solvents, in addition to being highly volatile, and may involve intensive and complicated synthetic procedures, which limit their application in solution-processed PSCs. Moreover, the vibrant colours displayed by some of the dopants may diminish the light absorption of the perovskite in the visible region of the spectrum [28]-[31]. Lithium bis(trifluoromethanesulfonyl)imide (Li-TFSI) is the most widely used $p$-type dopant and has been demonstrated to improve the conductivity of SpiroOMeTAD by shifting the Fermi level towards the highest occupied molecular orbital (HOMO) [15], [32]. The device efficiency is increased upon addition of Li-TFSI. This has been attributed to a reduction in the recombination losses at interfaces due to alteration of the $\mathrm{TiO}_{2}$ band edge position for higher potentials [9], [33]. However, the hygroscopic nature of Li-TFSI may result in formation of pin-holes in the Spiro-OMeTAD layer, thus promoting degradation of the underlying perovskite absorber [15], [34]-[36]. The addition of 4-tert butylpyridine (tBP) improves the miscibility of Spiro-OMeTAD and Li-TFSI by impeding phase segregation in solution, thus resulting in uniform distribution of Li-TFSI during thin film deposition. A drawback of tBP is its high volatility. Evaporation of the tBP leaves behind an accumulation of hygroscopic Li-TFSI [36], [37]. To address these key challenges, a simple technique for functionalization of Spiro-OMeTAD films has been investigated in this work based on a nonthermal atmospheric pressure plasma jet (APPJ) to increase the hole conductivity. 
Sterilization [38], [39], modification of polymers for improving surface wettability and cleaning [40]-[42], environmental, industrial [43] and medical applications [44] are few of the areas where non-thermal atmospheric pressure plasmas have been widely used. Plasma technology offers the advantage of 'dry doping' of semiconducting nanomaterials with heteroatoms to tune the band gap energy and conductivity for many promising applications. Atmospheric pressure plasma jets comprise charged particles, neutral metastable species, radicals and radiation in the UV and visible regions [45]. Non-thermal plasmas, also commonly known as 'cold plasmas' [46], [47], are characterized by a thermal non-equilibrium between the temperature of the electrons and the ions in the plasma jet such that the temperature of the electrons is a few electron volts but the temperature of the positively charged ions and neutral species is significantly lower (around room temperature). The near-ambient gas temperatures of non-thermal APPJs are suitable for treating temperature-sensitive materials. APPJs are also found to be compatible with conventional processes, and are easily adapted to complex geometries.

Oxygen plasmas have been explored extensively for surface functionalization of graphene and its oxide for use as electrodes in applications including light emitting diodes (LEDs), field effect transistors (FETs), and molecular sensors [48]-[50]. The application of plasma is mostly confined to surface cleaning and removal of impurities from fluorine doped tin oxide (FTO) and indium tin oxide (ITO) in dye-sensitized solar cells (DSSCs) and PSCs, improving device performance. However, recently the applications of plasma in PSCs have been widened to encompass improvement in device stability [51], surface modification [52], spray deposition of the perovskite active layer [53] and tuning the work function and surface energy of HTMs [54].

Other examples of the applications of non-thermal plasmas include: 
(i) Rolston et al. demonstrated a scalable, cold plasma spray technique for increasing stability of PSCs using a submicron organo-silicate barrier film [51]. Oxidizing species and heat from the plasma were reported to be responsible for the enhancement in device performance by improving interfacial contact and conductivity of the HTL.

(ii) A low-pressure Ar plasma treatment was used by Xiao et al. to modify the surface composition of the perovskite absorber by tuning the ratio of organic, inorganic components and defects [52]. This protocol resulted in an efficiency of $20.4 \%$ as a result of the enhancement of charge collection at the perovskite-electrode interface by impeding charge recombination.

(iii) A scalable, atmospheric pressure plasma, post-deposition technique to rapidly form an efficient and mechanically robust $\mathrm{CH}_{3} \mathrm{NH}_{3} \mathrm{PbI}_{3}$ perovskite layer without the need of a hot plate for curing the active material was reported by Hilt et al. [53]. An air plasma arc discharge was used for $250 \mathrm{~ms}$ exposure to obtain defect-free films showing ultrafast crystallization and a power conversion efficiency (PCE) of 15.7\%. Rapid nucleation and growth of perovskite crystals was monitored by in situ wide angle X-ray scattering and was attributed to the presence of reactive oxygen and nitrogen species in the plasma.

(iv) Oxygen plasma was used for tuning the surface energy and work function of hydrophobic polymeric HTMs such as P3HT [poly(3-n- hexylthiophene)], P3OT [Poly(3octylthiophene-2,5-diyl)], polyTPD [poly(N,N'-bis- 4-butylphenyl-N,N'bisphenyl)benzidine] and PTAA [poly(bis $\{4$-phenyl $\}\{2,4,6$-trimethylphenyl $\}$ amine $)]$ for applications as p-type contacts in inverted perovskite solar cell configuration, with a reported PCE over 19\% [54].

(v) Low-pressure oxygen plasma treatment on a $\mathrm{NiO}_{x}$ HTL layer in $\mathrm{p}-\mathrm{i}-\mathrm{n}$ architecture resulted in improved wettability and a consequent enhancement in efficiency to $12.3 \%$ [55]. 
(vi) Argon plasma functionalization was explored to not only increase the surface energy and roughness for an improved hydrophilicity and larger grain size of the perovskite film, but also to tune the conductivity by varying the ratio of PEDOT and PSS by plasma treatment [56].

(vii) Oxygen plasma at low-pressure has been demonstrated to rapidly oxidise Li-TFSI-doped Spiro-OMeTAD in PSCs with an enhancement in conductivity and mobility of the HTL [57]-[59]. Oxidation of Li-TFSI in Spiro-OMeTAD was assigned to the diffusion of oxidants generated in the plasma in the form of reactive oxygen and nitrogen species.

However, the studies focus on oxygen plasma generated at low pressure for functionalization of spiro-OMeTAD, which require a vacuum chamber, with its associated high maintenance, and high power for sustaining the discharge and are also not conducive with in-line large-scale processing technology. In the present study, a systematic and time-dependent, non-thermal atmospheric plasma treatment using a $\mathrm{He}-\mathrm{O}_{2} \mathrm{RF}-$ excited jet has been used to functionalize Spiro-OMeTAD films resulting in defect-free films with enhanced conductivity. The nonthermal APPJ used in this study being a non-abrasive technique, is suitable for temperaturesensitive materials. Moreover, Spiro-OMeTAD has a glass transition temperature of $125^{\circ}$ and a melting temperature of $248^{\circ}$ and when doped with Li-TFSI and tBP can sustain temperatures up to about $90^{\circ} \mathrm{C}$, above which degradation starts due to evaporation of the dopants [60]. The electronic and optical properties and surface morphology of Spiro-OMeTAD post timedependent non-thermal atmospheric plasma treatment are studied in detail.

\section{EXPERIMENTAL SECTION}

\section{Deposition of Spiro-OMeTAD films}


Thin films of Spiro-OMeTAD were deposited following the usual procedure reported elsewhere in the literature [61], [62]. In brief, $72 \mathrm{mg}$ of Spiro-OMeTAD was dissolved in $1 \mathrm{~mL}$ of chlorobenzene. $100 \mu \mathrm{L}$ of the solution was spin coated at $2000 \mathrm{rpm}$ onto Si wafer substrates (coupons measuring $1.5 \mathrm{~cm} \times 1.5 \mathrm{~cm}$ ) for 30 seconds. Prior to the deposition of thin films, the Si coupons were cleaned thoroughly by sequential ultra-sonication in acetone, deionized water and ethanol for 10 minutes each.

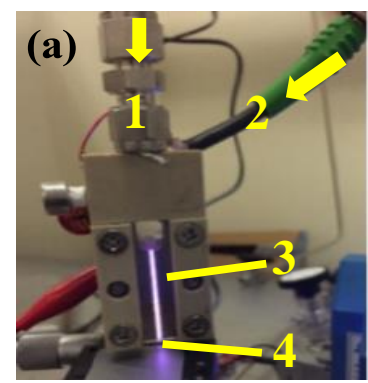

1: Gas flow

2: Radio frequency input

3: Electrodes

4: Plasma jet (b) He (3000 sccm) $+\mathrm{O}_{2}(30 \mathrm{sccm})$

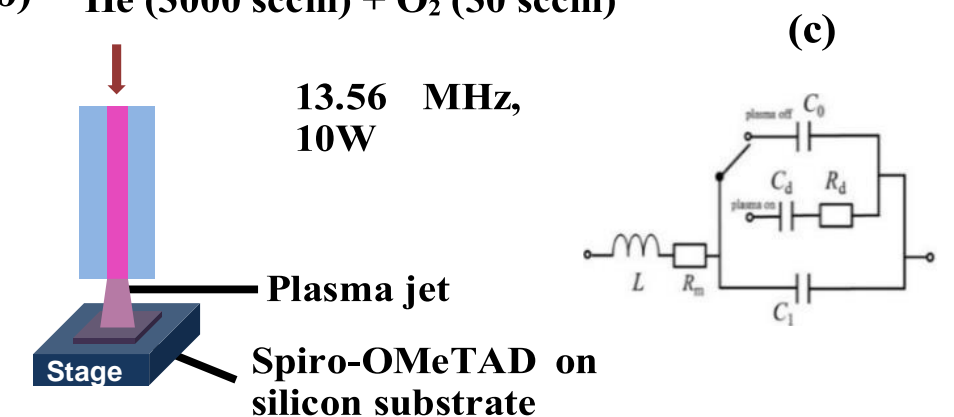

Figure 1(a) Experimental set-up of the RF atmospheric pressure plasma jet, (b) schematic of the $\mathrm{He}-\mathrm{O}_{2}$ plasma jet, and (c) the LC circuit with plasma ON condition.

\section{Atmospheric pressure plasma jet for surface functionalization}

The APPJ used for the functionalization of the Spiro-OMeTAD films is as shown in Figure 1(a); it is based on a precursor to the RF Reference Microplasma Jet [63], [64]. The plasma discharge is ignited between two plane-parallel stainless-steel electrode strips, $1 \mathrm{~mm}$ wide, $50 \mathrm{~mm}$ long, set $1 \mathrm{~mm}$ apart. The plasma is driven by applying radio frequency $(13.56 \mathrm{MHz})$ power through a series resonant LC circuit. The LC circuit consists of a tunable capacitor $\left(C_{\text {ext }}=2-22 \mathrm{pF}\right)$ and an inductor $(\mathrm{L}=16.5 \mu \mathrm{H})$ such that when the applied RF frequency matches that of the LC circuit, the voltage drop across the electrodes is amplified (resonance) by a factor of about 100, thus enabling the formation of a plasma discharge. 
With $\mathrm{He}$ as the carrier gas, the nominal input power required to sustain a plasma is typically $<10 \mathrm{~W}$. The flow rates of $\mathrm{He}$ and $\mathrm{O}_{2}$ used were 3000 and $30 \mathrm{sccm}$, respectively. Si coupons, preloaded with deposits of Spiro-OMeTAD, were placed at a distance of $5 \mathrm{~mm}$ from the plasma jet nozzle - far enough away to prevent the discharge transferring to the Si substrate, but close enough to encounter reactive species and radiation from the divergent plume which interacts with the surface over an area of $\sim 0.5 \mathrm{~cm} \times 0.5 \mathrm{~cm}$ that emanates from the electrode region. Our configuration is no more than few electrical watts in passing into the plasma. A fraction of that reaches the surface as the thermal energy of the gas plume (low heat capacity as gaseous and flow inherently cooling) plus internal energy on excited species that quench on surfacescomparable with thermal flux. Substrate surface therefore unlikely to be more than few tens this of degree above ambient- substrate bulk unlikely to be more than a few degrees above ambient depending on the mass and substructure. We have placed a thermocouple in contact with the substrate and temperature of the substrate was $37^{\circ} \mathrm{C}$. The specimens were rastered under the plume for 3, 4 and 5 minutes, with the ambition of obtaining uniformly functionalized thin films. During the process temperature of the substrate was measured to be 37 degrees Celsius. A minimum time of 3 minutes was used to allow for the homogeneous rastering of the available area of the pre-deposited films.

\section{Characterisation}

The characteristics of the plasma jet were studied via optical emission spectroscopy using an S2000+ Ocean Optics spectrometer.

The presence of oxidized Spiro-OMeTAD was confirmed by using UV-Vis spectroscopy with a JASCO UV-Vis equipment. 
X-ray photoelectron spectroscopy (XPS) was used to study the electronic properties with a load-locked KRATOS XSAM-800 instrument equipped with a dual anode X-ray source using an $\mathrm{Mg} \mathrm{K} \alpha(1253.6 \mathrm{eV})$ excitation source. The high magnification analyser mode was chosen to collect electrons from the smallest possible area on the specimen for $\mathrm{C} 1 s, \mathrm{O} 1 s$ and $\mathrm{N} 1 s$ core levels. CasaXPS software (version 2.3.16) was used to analyse the XPS data.

A ZEISS Supra 55 Variable Pressure Field Emission Scanning Electron Microscopy (SEM) equipment was used to study the morphology of the Spiro-OMeTAD films, before and after plasma treatment.

The thickness of the thin films was measured using the technique of Spectroscopic Ellipsometry with a Jobin-Yvon/Horiba equipment operating DeltaPsi2 v.2.0.8 software using linearly polarized light at $45^{\circ}$ incidence at three different positions on the thin films. The experimental wavelength range was chosen to be $250-800 \mathrm{~nm}(1.5-5.5 \mathrm{eV})$ and all measurements were made under ambient temperature, pressure and humidity conditions. The results were modelled using the Tauc-Lorentz oscillator model.

A Leica optical microscope was used to acquire white light images of the sample surfaces. An Asylum Research MFP3D AFM equipped with a Halcyonics vibration isolation stage was used to study the sample surfaces, mapping the surface conductivity and surface potential using Pt-Ir coated Si probes (PPP-EFM-10, Nanosensors) with a nominal force constant of $2.8 \mathrm{~N} \mathrm{~m}$ 1. The tip was biased at $+5 \mathrm{~V}$ with a drive amplitude of $300 \mathrm{mV}$. The image parameters were set at scan size of $10 \mu \mathrm{m} \times 10 \mu \mathrm{m}$, scan rate $0.5 \mathrm{~Hz}, 512$ points and lines. Electrostatic force microscopy (EFM) and Kelvin probe force microscopy (KPFM) measurements were performed in the 'tapping' mode and the 'Nap' mode at a delta height of $30 \mathrm{~nm}$ to obtain the height and potential profiles. The Gwyddion program was used to obtain the height profile and normalized nap phase images from the EFM and KPFM data. EFM is a dual-pass technique, where the 
topography is mapped first similar to the tapping mode AFM, followed by a repeated trace at a fixed delta height above the surface for measurement of the phase profile. Nap mode is performed with a conducting tip which is biased with a DC voltage. The phase and amplitude of vibration of the cantilever tip are affected when a voltage bias is applied between the tip and the sample surface. This type of imaging can be used to measure the relative change in the local electrostatic force between the tip and the sample surface. If the bias on the tip is kept constant along with the distance between the tip and the sample, then the measured variation in electrostatic force corresponds to the changes in surface potential distribution.

Sheet resistance was measured using a four-point probe technique and Keithley series, Model 2400 Source Meter. As the samples showed very high sheet resistance (order of G $\Omega$ sq1), the current range was carefully chosen at pA due to the sensitivity of the measurements. In this case, the current range was set at 50-100 pA and compliance at $210 \mathrm{~V}$. Attention was given to minimise the noise level that arises from the charging effect during data recording. The final value was averaged for a range of current values in pA to obtain higher accuracy.

\section{RESULTS AND DISCUSSION}

\section{Optical emission spectroscopy (OES) of the helium and oxygen plasma jet}

Optical emission spectroscopy provides a non-invasive probe to reveal the presence of specific atoms, ions and molecules within a plasma jet, thus providing insights into the chemical processes occurring in the plasma. The optical emission spectra of the effluent from the $\mathrm{He}-\mathrm{O}_{2}$ discharge into ambient air is shown in Figure 2. The gas phase composition, i.e. the excited atoms, ions and molecules determine the features in the spectra. Features from atomic and molecular oxygen, as well as helium and nitrogen species within the wavelength range of $200.0 \mathrm{~nm}$ to $880.0 \mathrm{~nm}$ is observed. Oxygen, being an electronegative element, its addition to 
the plasma leads to the formation of $\mathrm{O}_{-}, \mathrm{O}_{2-}$ ions and other species. Atomic and molecular lines of the reactive species are clearly observed in the spectra such as $\mathrm{OH}$ - transitions at $308.0 \mathrm{~nm}$, $\mathrm{N}_{2}$ (C-B) second positive system with electronic transitions $\mathrm{C}_{3} \prod_{\mathrm{u}} \rightarrow \mathrm{B}_{3} \prod_{\mathrm{g}}$ in the range 300$450 \mathrm{~nm}, \mathrm{He}$ I transition $3 \mathrm{~s}_{3} \mathrm{~S}_{1} \rightarrow 2 \mathrm{p}_{3} \mathrm{P}_{0}$ at $706.8 \mathrm{~nm}$, O I transitions $3 \mathrm{p}_{5} \mathrm{P} \rightarrow 4 \mathrm{~d}_{5} \mathrm{D}_{0}$ at $615.8 \mathrm{~nm}$, $3 \mathrm{p}_{5} \mathrm{P} \rightarrow 3 \mathrm{~s}_{5} \mathrm{~S}_{0}$ at $777.4 \mathrm{~nm}$ and $3 \mathrm{p}_{3} \mathrm{P} \rightarrow 3 \mathrm{~s}_{5} \mathrm{~S}_{0}$ at $844.8 \mathrm{~nm}$ and the A-band of molecular oxygen corresponding to the transition $\mathrm{b}_{1} \Sigma_{\mathrm{g}+} \rightarrow \mathrm{X}_{3} \Sigma_{\mathrm{g}-}$ at $760.0 \mathrm{~nm}[65]-[67]$. The spectral emission of molecular oxygen is generally very weak and hence, is not readily discernible in the emission spectra; in this work only the A-band at $760.0 \mathrm{~nm}$ has been recorded. Additional emission lines corresponding to $\mathrm{N}_{2+}$ first negative system with electronic transitions $\mathrm{B}_{2} \Sigma_{\mathrm{u}+} \rightarrow \mathrm{X}_{2} \Sigma_{\mathrm{g}+}(\Delta v=0)$ are observed at $391.3 \mathrm{~nm}$ and $427.0 \mathrm{~nm}$ [68]-[70]. Strong N2 emission lines in the spectrum arising from electron impact as the plasma plume propagates in ambient air were observed which has been reported previously for atmospheric pressure discharges in helium [43]. Interestingly, the emission line at $308.0 \mathrm{~nm}$ due to an $\mathrm{OH}$ - transition is very prominent in the He plasma jet but is suppressed in the $\mathrm{He}-\mathrm{O}_{2}$ jet, possibly due to the collisional quenching with oxygen molecules resulting in excited oxygen species in the plasma. The presence of $\mathrm{O} 777.4 \mathrm{~nm}$ emission suggests a preference for dissociative excitation whereas $\mathrm{O} 844.8 \mathrm{~nm}$ emission signifies the dominance of atomic oxygen excitation [66]. An increase in the intensity of both the $\mathrm{O}$ $777.4 \mathrm{~nm}$ and $\mathrm{O} 845 \mathrm{~nm}$ emission lines in the helium-oxygen plasma jet would suggest an interplay of both dissociative and direct excitation mechanisms. In the spectrum for a pure helium discharge, a series of strong peaks in the region of $215.0 \mathrm{~nm}$ to $271.0 \mathrm{~nm}$ originating from $\mathrm{NO}(\gamma) \mathrm{A}_{2} \Sigma_{+} \rightarrow \mathrm{X}_{2} \Pi$ is representative of the presence of oxygen in the afterglow [43], [71]. The reactive oxygen and nitrogen species in the plasma jet may include singlet oxygen, hydroxyl radicals, metastables and photons which play significant roles in the surface functionalization of Spiro-OMeTAD, as will be demonstrated in the present study. 

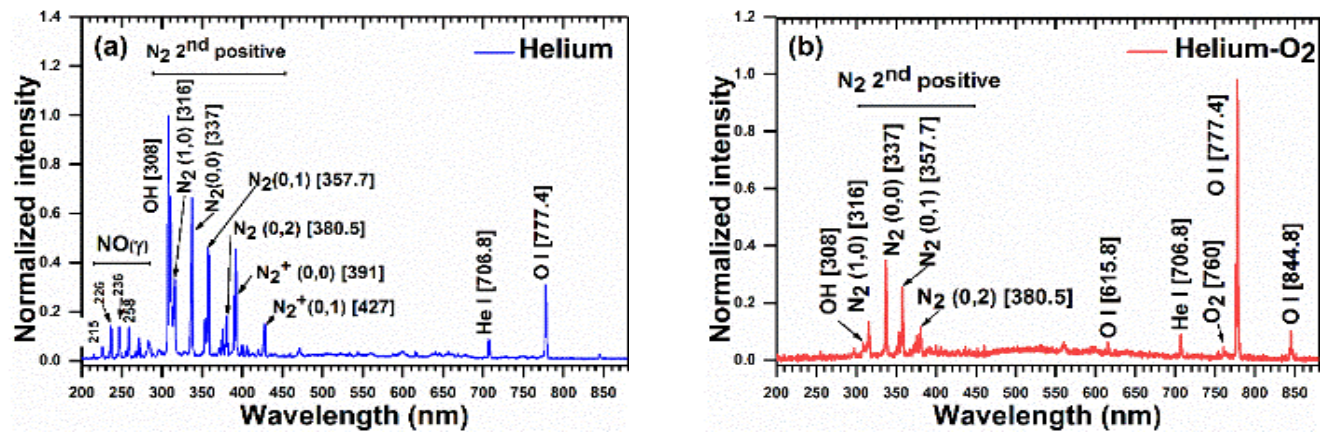

Figure 2 Optical emission spectra of the afterglow region of the APPJ: (a) He discharge, and (b) $\mathrm{He}(3000 \mathrm{sccm})+\mathrm{O}_{2}(30 \mathrm{sccm})$ discharge into ambient air.

\section{ELECTRONIC PROPERTIES}

$\mathrm{X}$-ray photoelectron spectroscopy (XPS) is an element-specific, site-selective technique which was used to probe the electronic properties and local chemical environment of the surface arising from plasma functionalization of the Spiro-OMeTAD films. C1s, N1s and O1s high resolution XPS spectra were energy calibrated with respect to the $\mathrm{C} 1 s$ of freshly cleaved highly oriented pyrolytic graphite (HOPG) at $284.5 \mathrm{eV}$ and $\mathrm{Au} 4 f$ peak at $84.0 \mathrm{eV}$ as reference. The pristine Spiro-OMeTAD molecule is composed of $81 \mathrm{C}, 4 \mathrm{~N}$ and $8 \mathrm{O}$ atoms. No X-ray induced damage was observed on the samples. 


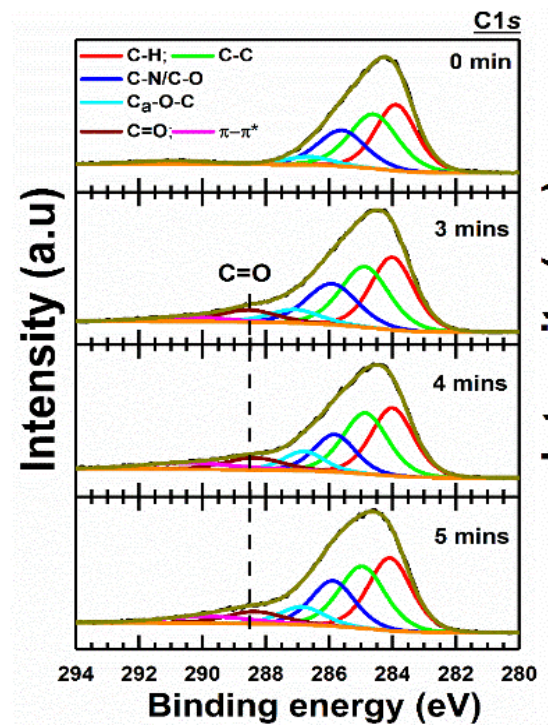

(a)

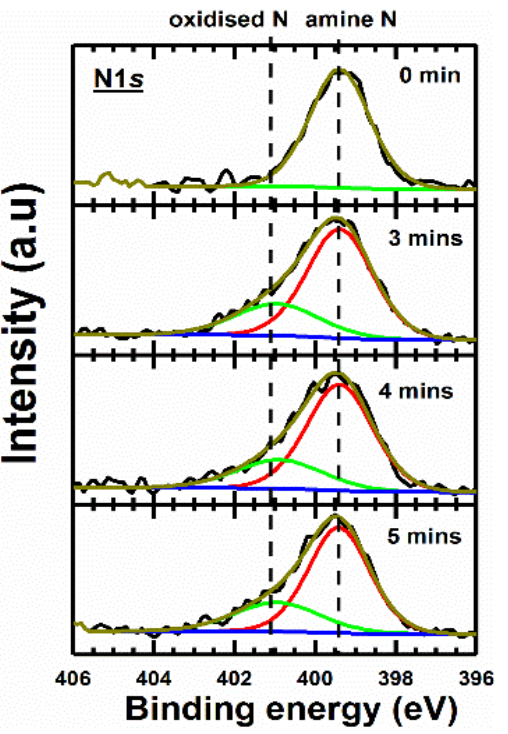

(b)

Figure 3 Variation of (a) C1s and (b) N1s XPS spectra of Spiro-OMeTAD as a function of plasma treatment time showing the appearance of bound-oxygen features.

C1s: The $\mathrm{C} 1 s$ core level spectra as a function of plasma treatment time is shown in Figure 3(a). The $\mathrm{C} 1 s$ spectra were fitted with an asymmetric Gaussian-Lorentzian line shape. Results of the fitting and deconvolution process indicates that untreated Spiro-OMeTAD is composed of C$\mathrm{H}, \mathrm{C}=\mathrm{C} / \mathrm{C}-\mathrm{C}, \mathrm{C}-\mathrm{N}, \mathrm{C}-\mathrm{O}-\mathrm{C}$ and $\pi-\pi^{*}$ components. The main peak at $283.9 \pm 0.1 \mathrm{eV}$ corresponds to $\mathrm{C}-\mathrm{H}$ functionality, followed by the contribution at $284.8 \pm 0.1 \mathrm{eV}$ which is from a combined effect of $\mathrm{C}=\mathrm{C}$ and $\mathrm{C}-\mathrm{C}$ groups. The $s p 3$ bonded carbon occurs at $0.5 \mathrm{eV}$ above the binding energy of $s p 2$ bonded configuration. The components at $285.8 \pm 0.1 \mathrm{eV}$ and $286.9 \pm 0.2 \mathrm{eV}$ have contributions from carbon singly bonded to nitrogen and $\mathrm{C}_{\mathrm{a}}-\mathrm{O}-\mathrm{C}(\mathrm{C}$ a signifies the aromatic carbon), respectively [16], [23], whereas the feature at $\sim 291.0 \mathrm{eV}$ stems from $\pi-\pi^{*}$ plasmon contribution. The values agree quite well (within $\pm 0.5 \mathrm{eV}$ ) with previous literature.

The comparison of $\mathrm{C} 1 s$ line shape of untreated and plasma-treated Spiro-OMeTAD in Figure 3(a) shows a significant inhomogeneous broadening towards higher binding energy after 3 minutes of plasma treatment. This broadening can be attributed to the surface oxidation of 
Spiro-OMeTAD films via the attachment of functional groups such as $\mathrm{C}-\mathrm{OH}$ and $\mathrm{C}=\mathrm{O}$ [72], [73]. The $\mathrm{C} 1 s$ spectra of the plasma-functionalized films were deconvoluted into $\mathrm{C}-\mathrm{H}, \mathrm{C}=\mathrm{C} / \mathrm{C}-$ $\mathrm{C}, \mathrm{C}-\mathrm{N}, \mathrm{Ca}-\mathrm{O}-\mathrm{C}, \mathrm{C}=\mathrm{O}$ and $\pi-\pi^{*}$ components. The additional $\mathrm{C}=\mathrm{O}$ component at $288.5 \mathrm{eV}$ is due to the presence of different oxidized species in the plasma jet which bonded to carbon atoms of the Spiro-OMeTAD film. The bombardment of the energetic ionic species present in the plasma jet may lead to the formation of defects which in turn form favourable sites for the attachment of oxygen moieties and hence, contribute to surface functionalization. The fitting parameters extracted from the XPS spectra are summarized in Table 1.

From Figure 3(a), a $0.2 \mathrm{eV}$ decrease in the FWHM of the $\mathrm{C}$ bonded to $\mathrm{C}$ component from 1.8 (pristine) to $1.6 \mathrm{eV}$ (4 minutes) is observed. Though the resolution of the XPS equipment is $0.9 \mathrm{eV}$, careful fitting of the line shape of $\mathrm{C} 1 s$ with a combination of Gaussian-Lorentzian line shapes was undertaken to interpret the FWHM variation in the spectra. The increase in concentration of aromatic $\mathrm{C}$ bonded to $\mathrm{O}(\mathrm{Ca}-\mathrm{O}-\mathrm{C})$ component from $4.9 \%$ (pristine) to $7.0 \%$ upon 3 minutes of plasma functionalization is followed by a further enhancement to $8.5 \%$ and $7.9 \%$ after 4 minutes and 5 minutes of plasma exposure, respectively. A monotonic increase in the $\mathrm{C}=\mathrm{O}$ component is also observed from 0 to $7.0 \%$. This signifies an enhancement in the carbon bonded to oxygen species to the aromatic structure of Spiro-OMeTAD molecule. Figure 4 shows the trend of increasing (a) $\mathrm{Ca}_{\mathrm{a}}-\mathrm{O}-\mathrm{C}$ and (b) $\mathrm{C}=\mathrm{O}$ species from pristine to functionalized Spiro-OMeTAD samples. A steady increase in the area of the $\pi$ - $\pi^{*}$ plasmon peak is also discernible upon plasma functionalization from $3.6 \%$ (pristine) to $6.4 \%$ after 4 minutes of plasma treatment before settling at $6.0 \%$ after 5 minutes of exposure.

The aromaticity of Spiro-OMeTAD molecules can be described in terms of the FWHM of the $\mathrm{C}-\mathrm{C} / \mathrm{C}=\mathrm{C}$ component and the area of the plasmon feature, where a larger $\pi-\pi^{*}$ peak would signify a polyaromatic system [74], [75]. Hence, in this study, the reduction in FWHM of the 
C-C component and the increase in area of $\pi-\pi^{*}$ component can be attributed to an improvement in aromaticity of Spiro-OMeTAD upon functionalization using the atmospheric pressure He$\mathrm{O}_{2}$ plasma jet. Increase in aromaticity might contribute to the increase in conductivity discussed in later sections.

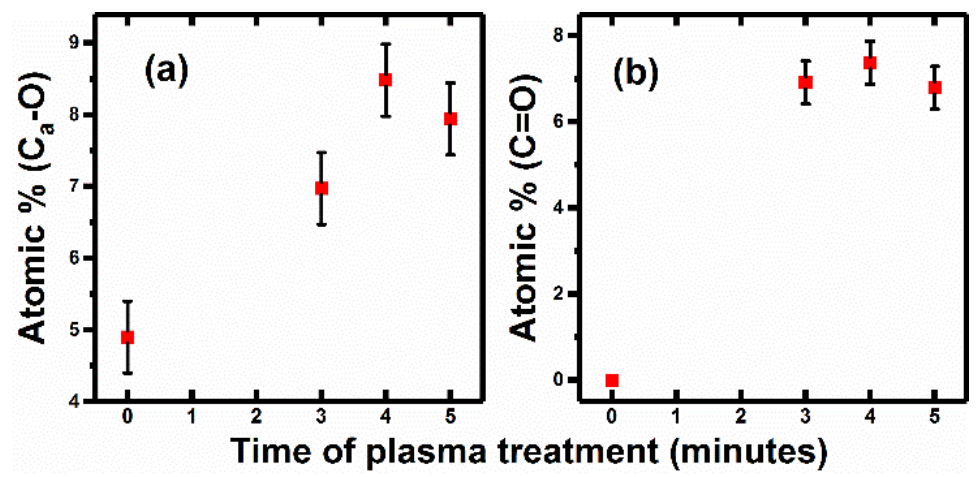

Figure 4 Variation of atomic percentage of (a) $\mathrm{C}_{\mathrm{a}}-\mathrm{O}-\mathrm{C}$ and (b) $\mathrm{C}=\mathrm{O}$ component with time of plasma functionalization.

N1s: High resolution N1s spectra of untreated and time-dependent plasma-treated SpiroOMeTAD have been compared in Figure 3(b). There is ambiguity in the literature with regard to assignment of the exact position of the amine $\mathrm{C}-\mathrm{N}$ in pristine Spiro-OMeTAD, with Scholin et al. having reported the $\mathrm{N} 1 s$ peak due to amine $\mathrm{C}-\mathrm{N}$ from Spiro-OMeTAD at $400.3 \mathrm{eV}$ from XPS studies on a similar triphenylamine hole conductor molecule [76], [77]. However, in this study the spectrum for the untreated Spiro-OMeTAD film was fitted with one component centred at $\sim 399.5 \pm 0.15 \mathrm{eV}$ corresponding to the amine $\mathrm{N}$ species of Spiro-OMeTAD [16], [78]-[81] which is $\sim 0.8 \mathrm{eV}$ shifted from other reports. The spectra for the plasma-treated films, irrespective of the time of plasma functionalization, show a substantial broadening towards the higher binding energy and are fitted with an additional component centred at $\sim 401.0 \pm 0.2 \mathrm{eV}$. The higher binding energy component may have a contribution from the oxidized $\mathrm{N}$ species $[82]-[84]$. 
A slight variation in the FWHM of the amine $\mathrm{N}$ component which has been fitted with a FWHM of $1.8 \pm 0.15 \mathrm{eV}$ is observed with increased time of plasma exposure. Figure 5 shows the variation of FWHM of oxidized N component in the fitted N1s spectra with respect to time of plasma exposure (0, 3, 4 and 5 minutes). An increase in FWHM of the oxidized N component to $2.5 \mathrm{eV}$ upon plasma-exposure has been observed with pristine Spiro-OMeTAD showing no oxidized $\mathrm{N}$ component (Figure $3 \mathrm{~b}$ ). The amine $\mathrm{N}$ is thought to be the active site for the attachment of Li-TFSI dopant molecules in doped Spiro-OMeTAD [85]. It has been proposed here that plasma modifies the amine $\mathrm{N}$ site and the formation of the oxidized nitrogen species may result in improved conductivity of the films post-treatment as discussed in later sections. Loss in methyl groups as a result of plasma-spiro-OMeTAD interaction may result in formation of quinoid structures with quaternary nitrogen $+\mathrm{N}=\mathrm{C}$ which can also contribute to the enhancement in positive charges in the molecule.

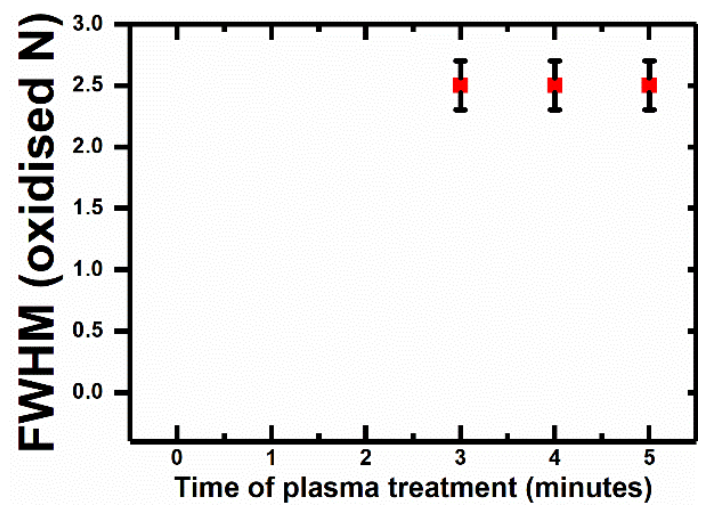

Figure 5 Variation of FWHM of oxidized N component in fitted N1s spectra with respect to time of plasma treatment (0, 3, 4 and 5 minutes).

Though a variation in the nitrogen environment has been observed from the $\mathrm{N} 1 s$ spectra, no such change is observed in the C-N component of the $\mathrm{C} 1 s$ spectra since it is overlapped with the contribution from the C-O component and cannot be deconvoluted due to the $0.9 \mathrm{eV}$ resolution of the XPS equipment. 
O1s: The O1s core level spectrum of the as-deposited film was fitted with one component centred at $\sim 533.3 \pm 0.1 \mathrm{eV}$ corresponding to the $\mathrm{C}_{\mathrm{a}-\mathrm{O}} \mathrm{O}$ as reported previously in the literature [16], [18]. After plasma treatment, pristine Spiro-OMeTAD films show a $0.5 \mathrm{eV}$ increase in FWHM (Figure 6). This $0.5 \mathrm{eV}$ increase signifies an increased degree of oxidation of the SpiroOMeTAD films with time of plasma exposure. This broadening can be attributed to the formation of $\mathrm{O}$ doubly bonded to $\mathrm{C}$ at $\sim 531.1 \pm 0.1 \mathrm{eV}$ [73], [86], [87] which can be corroborated by the $\mathrm{C}=\mathrm{O}$ signature from the $\mathrm{C} 1$ s spectra of plasma-functionalized SpiroOMeTAD, shown in Figure 3 (a). The formation of dangling bonds upon plasma-surface interaction have the tendency to saturate with the formation of $\mathrm{C}=\mathrm{O}$ species since $\mathrm{C}=\mathrm{O}$ is stable and energetically favourable. The $\mathrm{C}=\mathrm{O}$ component may have a contribution either from carbonyl or carboxylic groups. The formation of carboxylic group upon plasma exposure can be used to explain the increase in conductivity of functionalized Spiro-OMeTAD by improving the flexibility of the aromatic backbone or promoting charge carriers along the molecule [88]. The variation of FWHM of the $\mathrm{Ca}_{\mathrm{a}} \mathrm{O}$ component in fitted $\mathrm{O} 1 s$ spectra with respect to plasma treatment time is shown in Figure 7. A monotonic increase in the FWHM is observed with increase in time of plasma exposure.

Surface oxidation is consistent with the positive variation in work function measured with KPFM which proves the incorporation of oxygen functional groups on the surface. Though chemical oxidation is the most common doping strategy for HTLs, other techniques have also been explored such as acidic dopants for conductive polymers, where the enhancement in conductivity is attributed to the induction of positive charges that increase the carrier density in the systems [89]. The aforementioned quinoid structures may also be responsible for the increase in positive charge carriers. It is also proposed that oxygen plasma induces an increase in positive charges in Spiro-OMeTAD that enhances the carrier density and hence, the improvement in conductivity. 


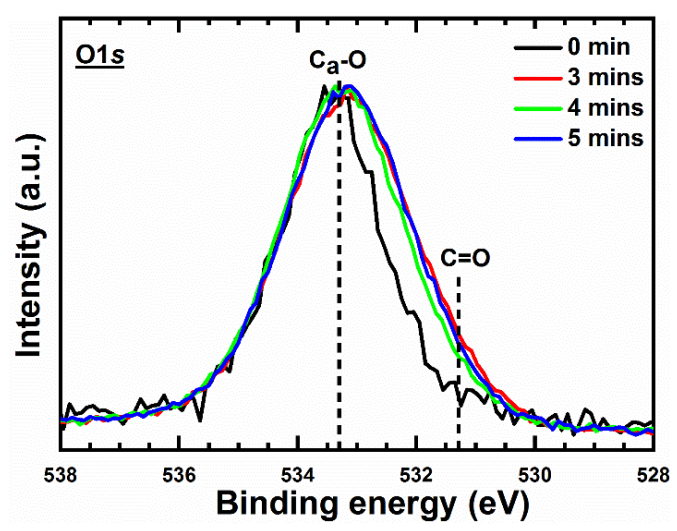

Figure 6 Variation of O1s XPS spectra of Spiro-OMeTAD with respect to plasma treatment time (0, 3, 4 and 5 minutes).

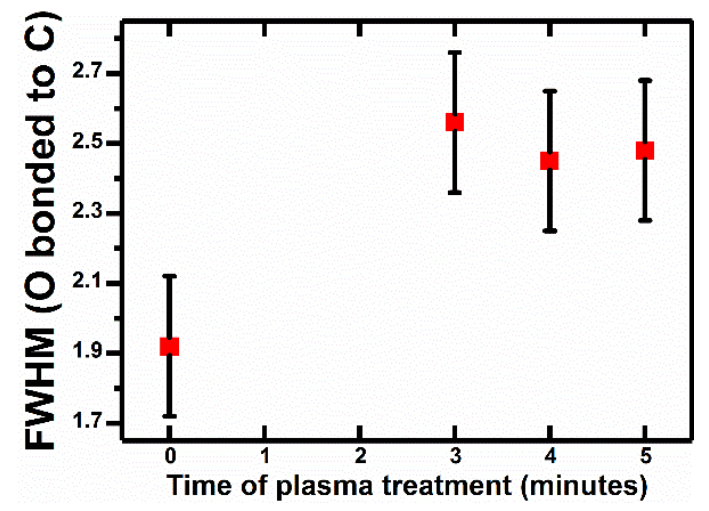

Figure 7 Variation of FWHM of the $\mathrm{C}$ bonded to $\mathrm{O}(\mathrm{C}=\mathrm{O} / \mathrm{C}-\mathrm{O}-\mathrm{C})$ in fitted $\mathrm{O} 1 s$ spectra with respect to plasma treatment time $(0,3,4$ and 5 minutes $)$.

Table 1 Fitting parameters extracted from XPS spectra of pristine and $\mathrm{He}+\mathrm{O}_{2}$ plasma functionalized Spiro-OMeTAD.

\begin{tabular}{|c|c|c|c|c|}
\hline Sample & Region & Component & \% conc. $\pm \mathbf{0 . 2}$ & FWHM $\pm \mathbf{0 . 1}$ eV \\
\hline \multirow{4}{*}{} & $\mathrm{C} 1 s$ & $\mathrm{C}-\mathrm{H}$ & 35.0 & 1.5 \\
\cline { 3 - 5 } & & $\mathrm{C}=\mathrm{C} / \mathrm{C}-\mathrm{C}$ & 34.0 & 1.8 \\
\cline { 3 - 5 } & $\mathrm{C}-\mathrm{N} / \mathrm{C}-\mathrm{O}$ & 22.2 & 1.7 \\
\cline { 3 - 5 } & $\mathrm{Ca}-\mathrm{O}-\mathrm{C}$ & 5.0 & 1.7 \\
\cline { 3 - 5 } $\begin{array}{c}\text { Untreated Spiro- } \\
\text { OMeTAD }\end{array}$ & & $\mathrm{C}=\mathrm{O}$ & 0 & 0 \\
\cline { 3 - 5 } & & $\pi-\pi^{*}$ & 3.6 & 2.8 \\
\hline
\end{tabular}




\begin{tabular}{|c|c|c|c|c|}
\hline & $\mathrm{O} 1 s$ & $\mathrm{Ca}-\mathrm{O}-\mathrm{C}$ & 100.0 & 2.0 \\
\hline & $\mathrm{N} 1 s$ & amine $\mathrm{N}$ & 100.0 & 1.7 \\
\hline & & Oxidized N & 0 & 0 \\
\hline \multirow{9}{*}{$\begin{array}{c}3 \text { mins plasma } \\
\text { Spiro-OMeTAD }\end{array}$} & \multirow[t]{6}{*}{$\mathrm{C} 1 s$} & $\mathrm{C}-\mathrm{H}$ & 31.6 & 1.6 \\
\hline & & $\mathrm{C}=\mathrm{C} / \mathrm{C}-\mathrm{C}$ & 29.7 & 1.9 \\
\hline & & $\mathrm{C}-\mathrm{N} / \mathrm{C}-\mathrm{O}$ & 21.0 & 1.9 \\
\hline & & $\mathrm{Ca}_{\mathrm{a}} \mathrm{O}-\mathrm{C}$ & 7.0 & 1.9 \\
\hline & & $\mathrm{C}=\mathrm{O}$ & 6.9 & 2.1 \\
\hline & & $\pi-\pi^{*}$ & 3.6 & 2.9 \\
\hline & $\mathrm{O} 1 s$ & $\mathrm{Ca}-\mathrm{O}-\mathrm{C}$ & 100.0 & 2.6 \\
\hline & $\mathrm{N} 1 s$ & amine $\mathrm{N}$ & 72.6 & 1.9 \\
\hline & & Oxidized N & 27.3 & 2.5 \\
\hline \multirow{9}{*}{$\begin{array}{c}4 \text { mins plasma } \\
\text { Spiro-OMeTAD }\end{array}$} & \multirow[t]{6}{*}{$\mathrm{C} 1 s$} & $\mathrm{C}-\mathrm{H}$ & 31.3 & 1.6 \\
\hline & & $\mathrm{C}=\mathrm{C} / \mathrm{C}-\mathrm{C}$ & 29.7 & 1.7 \\
\hline & & $\mathrm{C}-\mathrm{N} / \mathrm{C}-\mathrm{O}$ & 16.6 & 1.5 \\
\hline & & $\mathrm{Ca}-\mathrm{O}-\mathrm{C}$ & 8.5 & 1.5 \\
\hline & & $\mathrm{C}=\mathrm{O}$ & 7.4 & 2.1 \\
\hline & & $\pi-\pi^{*}$ & 6.4 & 3.5 \\
\hline & $\mathrm{O} 1 s$ & $\mathrm{Ca}-\mathrm{O}-\mathrm{C}$ & 100.0 & 2.5 \\
\hline & $\mathrm{N} 1 s$ & amine $\mathrm{N}$ & 73.6 & 1.9 \\
\hline & & Oxidized $\mathrm{N}$ & 26.4 & 2.5 \\
\hline \multirow{9}{*}{$\begin{array}{l}5 \text { mins plasma } \\
\text { Spiro-OMeTAD }\end{array}$} & \multirow{6}{*}{$\mathrm{C} 1 s$} & $\mathrm{C}-\mathrm{H}$ & 31.2 & 1.6 \\
\hline & & $\mathrm{C}=\mathrm{C} / \mathrm{C}-\mathrm{C}$ & 28.4 & 1.7 \\
\hline & & C-N/C-O & 19.6 & 1.6 \\
\hline & & $\mathrm{C}_{\mathrm{a}}-\mathrm{O}-\mathrm{C}$ & 8.0 & 1.6 \\
\hline & & $\mathrm{C}=\mathrm{O}$ & 6.8 & 2.1 \\
\hline & & $\pi-\pi^{*}$ & 6.0 & 3.3 \\
\hline & $\mathrm{O} 1 s$ & $\mathrm{C}_{\mathrm{a}}-\mathrm{O}-\mathrm{C}$ & 100.0 & 2.5 \\
\hline & $\mathrm{N} 1 s$ & amine $\mathrm{N}$ & 72.0 & 1.8 \\
\hline & & Oxidized N & 28.0 & 2.5 \\
\hline
\end{tabular}

\section{Morphology of Spiro-OMeTAD films}

The morphology of the Spiro-OMeTAD films before and after time-dependent plasma treatment was studied using optical microscopy, scanning electron microscopy (SEM) and atomic force microscopy (AFM). No evidence of pinholes was observed in the optical, SEM and AFM images. 
There was no noticeable difference in film morphology before and after plasma treatment when studied using the SEM (Figure S1, supporting information). The films were appreciably uniform, except for the presence of silicon dust on the surfaces (observed as small dots in the figure). As was visible with the naked eye, the change in colour from the as-deposited film to the plasma-functionalized film, was corroborated with optical microscopy (Figure S2, supporting information). The change in colour of the films post-treatment from greenish to bluish became discernible as the time of plasma exposure was increased to 4 and 5 minutes.
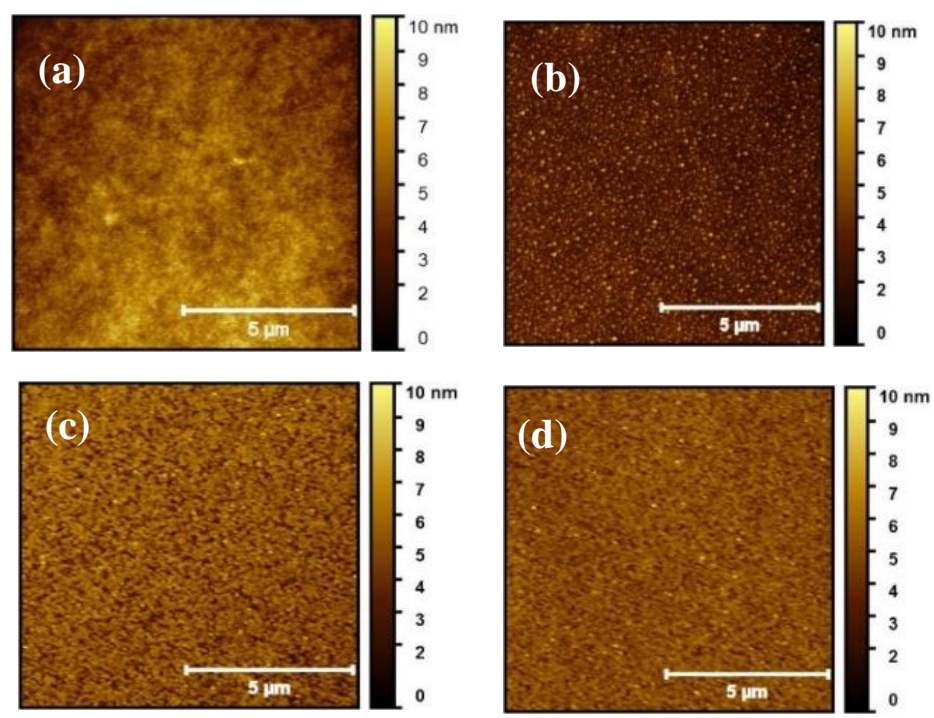

Figure 8 AFM images of (a) untreated and (b, c, d) plasma-treated Spiro-OMeTAD with 3, 4 and 5 minutes of exposure time.

Figure 8 shows the AFM height profiles of the pristine and plasma-treated Spiro-OMeTAD films. Subtle variation in film roughness was noticeable with films post-treatment showing enhanced $R_{r m s}$ (rms roughness values) from $0.8 \mathrm{~nm}$ to $1.2 \mathrm{~nm}$, though some accumulation of aggregates on the surface is seen to increase with increased time of plasma exposure. This indicates that the low power plasma jet used in the current work does not induce noticeable surface damage to the Spiro-OMeTAD films. The thickness of the untreated Spiro-OMeTAD 
film obtained from ellipsometry measurements was $139.2 \mathrm{~nm}$ and after 5 minutes of plasma treatment, the value was $140.5 \mathrm{~nm}$. The thickness values are an average of measurements from three different positions on the films. The subtle difference between the thickness of plasmatreated and untreated films signifies that the APPJ is not an abrasive technique and does not result in corrosion of the surface of Spiro-OMeTAD.

\section{Optical properties - UV-vis spectroscopy}

The UV-vis absorption spectra of the pristine and plasma treated Spiro-OMeTAD are shown in Figure 9. Spiro-OMeTAD has a sharp absorption peak in the UV region at around $395.0 \mathrm{eV}$ [90]. But when oxidized, there is an absorption peak at $400.00 \mathrm{~nm}$, along with an additional broad band at around $500.00 \mathrm{~nm}$ which is the signature of the Spiro-OMeTAD+ radical [91]. However, the $400.00 \mathrm{~nm}$ peak is often indistinguishable and overlapped with the strong peak at $395.00 \mathrm{~nm}$ corresponding to non-oxidized Spiro-OMeTAD. From the figure, differences in the UV-vis spectra of plasma-treated and untreated films are clearly discernible. The absorption peak at $\sim 480.0 \mathrm{~nm}$ due to the presence of the oxidized Spiro-OMeTAD radical in the plasma functionalized films is absent in the undoped film. This proves that the Spiro-OMeTAD molecule has become oxidized after plasma exposure. The absorption peak at $\sim 395.0 \mathrm{~nm}$ which is due to pristine Spiro-OMeTAD slowly decreases in intensity with increase in time of plasma exposure for 3 minutes and 4 minutes of exposure time but an increase is observed after 5 minutes of plasma functionalization which might have a contribution from the $400 \mathrm{~nm}$ peak of oxidized Spiro-OMeTAD due to the prolonged time of plasma exposure. The absorption peak at $\sim 480.0 \mathrm{~nm}$ due to the oxidized Spiro-OMeTAD radical in the plasma functionalized films increases initially for 3 minutes of treatment time before decreasing upon prolonged exposure. But the decrease in intensity is accompanied by a broadening of the peak which might have a 
contribution from the different degree of oxidation of the plasma-treated films with treatment time.

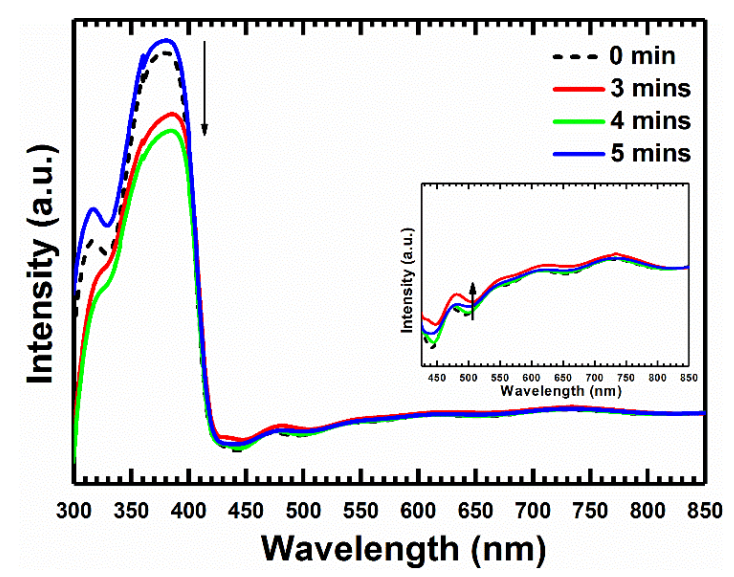

Figure 9 UV-vis spectra of pristine and plasma-functionalized Spiro-OMeTAD.

\section{Electrical properties}

\section{EFM and 4-point probe sheet resistance}

Electrostatic force microscopy is a useful technique for investigating the electrical properties of nanomaterials and has been extensively used to study the distribution of electrical potential and charge at the nanoscale for graphene and graphene oxide [92]-[95]. Qualitative mapping of the surface potential is primarily undertaken with EFM. Though not widely exploited in the field of organic-inorganic hybrid halide PSCs, Juarez-Perez et al. used EFM to investigate the role of chemical dopants, Li-TFSI and tBP, on the morphological and transport properties of Spiro-OMeTAD [61]. They mapped the distribution of dopants on the Spiro-OMeTAD film and the role of tBP as a homogenizer preventing phase separation of Spiro-OMeTAD and LiTFSI in solution was established. 
In the present work, EFM has been used to evaluate the electrical properties of SpiroOMeTAD thin films at the nanometre scale before and after plasma treatment. EFM measurements were carried out around the same area of the sample before and after 3, 4 and 5 minutes of plasma exposure. In EFM, the capacitive force between a biased tip and the grounded sample surface induces phase shifts of the tip oscillations given by the following equation [96], [97]

$\Delta \varphi=\frac{Q}{2 k} \frac{d^{2} C}{d z^{2}}\left(V_{t}-\varphi\right)^{2}$

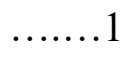

In equation $1, \Delta \varphi$ is the phase shift, $Q$ and $k$ are the quality factor and spring constant of the cantilever, $C$ is the capacitance of the system, $V_{t}$ is the voltage of the tip, $z$ is the distance between the Pt-Ir tip and sample, and $\phi$ is the surface potential. A greater contrast in the pseudo colour image would indicate increased fluctuations in the phase value and signifies a region of lower surface conductivity, whereas a darker colour would mean lower phase shift and hence, a higher surface conductivity.

The pseudo colour phase images before plasma treatment for all the samples show a uniform and high phase value as in Figure $10(\mathrm{a}, \mathrm{c}, \mathrm{e})$, whereas after 3 minutes of plasma exposure, the phase values reduce and the formation of small blob-like structures were observed in the height profiles (Figure 8b). Interestingly, these blobs or accumulations which are fairly uniformly distributed over the surface of the plasma-functionalized films are the locations with lower phase or higher conductivity values. The reduction in phase value would signify an improvement in the surface conductivity of the samples after plasma functionalization. After longer plasma exposure of 4 and 5 minutes, no blob-like structures were visible in the height images, though the overall pseudo colour images showed a trend towards lower phase values. The average value of the phase shift over the measured area was observed to have reduced by $0.20^{\circ}, 0.46^{\circ}$ and $0.20^{\circ}$ after plasma treatment for 3,4 and 5 minutes, respectively, as 
summarized in Table 2. Thus, it can be concluded that the APPJ has resulted in improvement of the surface conductivity of the samples. The surface roughness of the films after plasma exposure has undergone a slight increase as is evident from the roughness values.
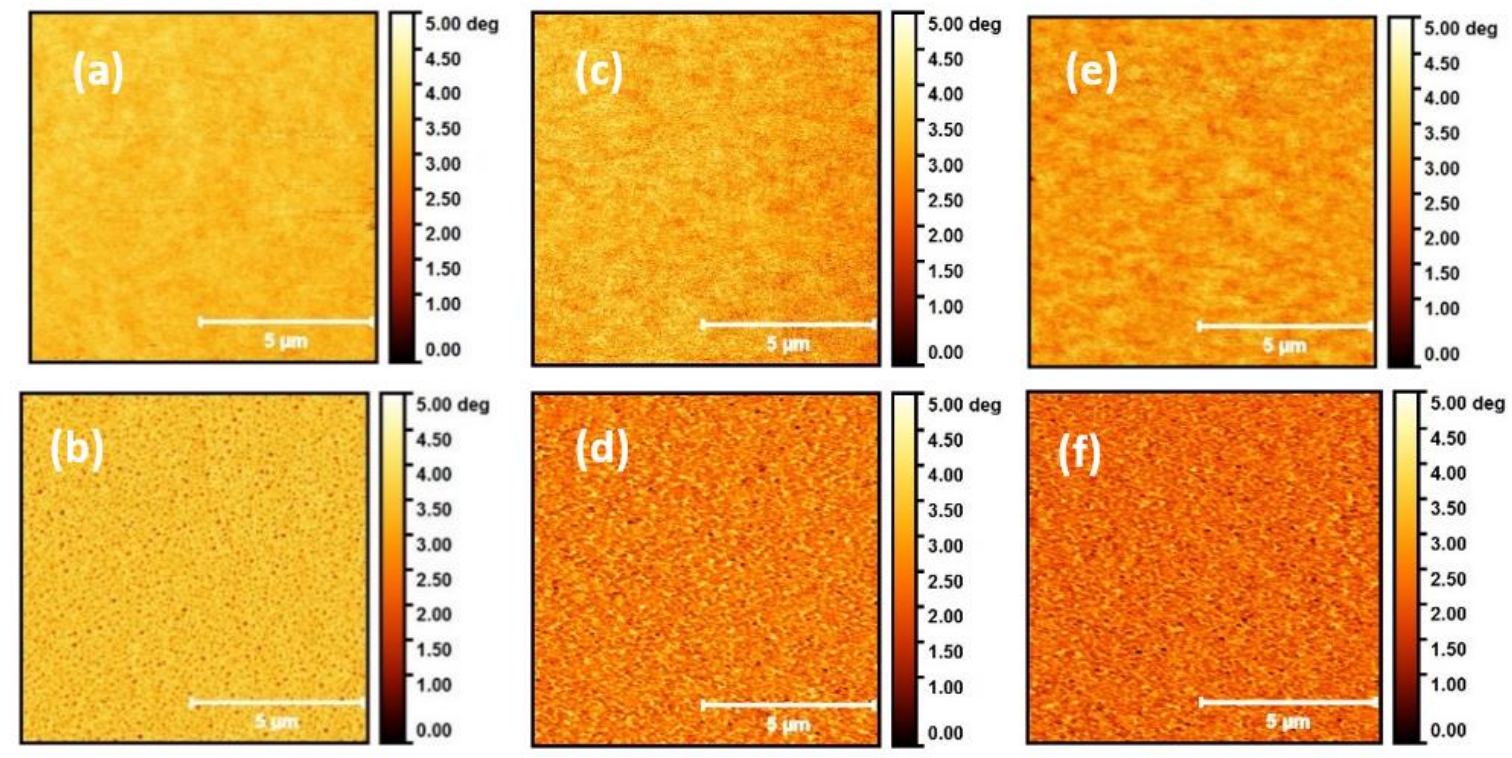

Figure 10 EFM phase images of pristine (a, c, e) and samples treated with plasma for 3, 4 and 5 mins, respectively (b, d, f). A greater contrast in the pseudo colour image shows increased fluctuations in the phase value and signifies a less conducting region, whereas a darker colour means lower phase shift and hence more surface conducting nature of sample.

Table 2 Variation of the average phase before and after plasma treatment of Spiro-OMeTAD.

\begin{tabular}{|c|c|c|c|}
\hline Samples & $\begin{array}{c}\text { Avg. phase (Pre-treatment) } \\
\left({ }^{\circ}\right)\end{array}$ & $\begin{array}{c}\text { Avg. phase (Post-treatment) } \\
\left({ }^{\circ}\right)\end{array}$ & $\begin{array}{c}\text { Change in } \\
\text { phase }\left({ }^{\circ}\right)\end{array}$ \\
\hline 3 mins & 3.6 & 3.4 & -0.20 \\
\hline
\end{tabular}




\begin{tabular}{|c|c|c|c|}
\hline 4 mins & 3.0 & 2.54 & -0.46 \\
\hline $5 \mathrm{mins}$ & 2.89 & 2.69 & -0.2 \\
\hline
\end{tabular}

Table 3 Variation of the sheet resistance values and conductivity of pristine and plasma-treated Spiro-OMeTAD.

\begin{tabular}{|c|c|c|c|c|c|}
\hline & Sample ID & $\begin{array}{l}\text { Period of } \\
\text { plasma } \\
\text { treatment } \\
\text { (mins) }\end{array}$ & $\begin{array}{c}\text { Sheet } \\
\text { resistance } \\
\text { (G } \Omega \text { sq-1) }\end{array}$ & $\begin{array}{c}\text { Thickness } \\
\text { (nm) }\end{array}$ & $\begin{array}{l}\text { Conductivity } \\
\text { (S cm-1) }\end{array}$ \\
\hline 1 & $\begin{array}{c}\text { Untreated Spiro- } \\
\text { OMeTAD }\end{array}$ & 0 & 80.5 & 139.2 & $9.40 \times 10-7$ \\
\hline 2 & \multirow{3}{*}{$\begin{array}{l}\text { Plasma-treated } \\
\text { Spiro-OMeTAD }\end{array}$} & 3 & 11.3 & 139.7 & $6.36 \times 10-6$ \\
\hline 3 & & 4 & 8.0 & 158.8 & $7.80 \times 10-6$ \\
\hline 4 & & 5 & 6.2 & 140.5 & $1.15 \times 10-5$ \\
\hline
\end{tabular}
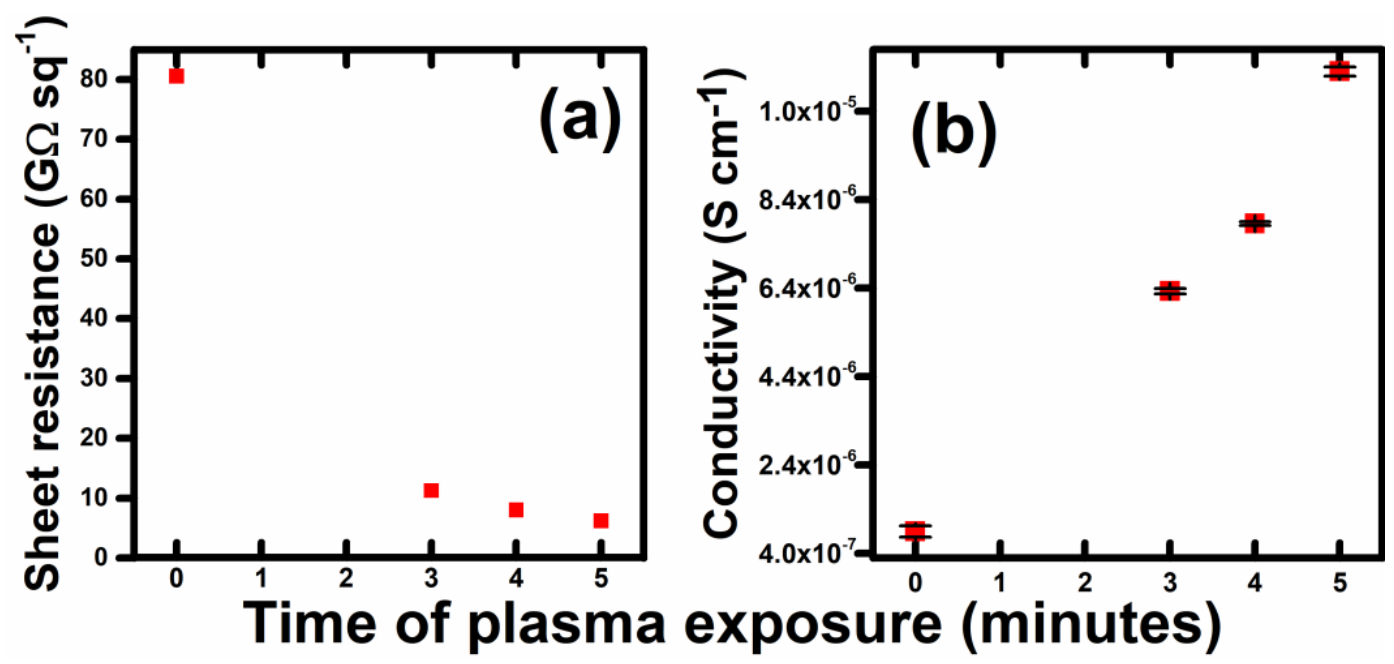
Figure 11 Variation of (a) sheet resistance and (b) conductivity of Spiro-OMeTAD with time of plasma functionalization.

Oxygen plasma treatment results in functionalization of the surface of thin films by incorporation of polar functional groups such as C-O, C-OH [98], [99] which may lead to enhancement of the conductivity of the films. This was also supported by the conductivity values of the samples calculated from the measured sheet resistance before and after the plasma functionalization as shown in Table 3. Pristine Spiro-OMeTAD films showed a conductivity value of $9.4 \times 10_{-7} \mathrm{~S} \mathrm{~cm}-1$ which agrees well with previous reports [13]. The conductivity increased by an order of magnitude to $6.36 \times 10_{-6} \mathrm{~S} \mathrm{~cm}-1$ after 3 minutes of plasma treatment which is comparable to reported values of doped Spiro-OMeTAD with $p$-type dopant Li-TFSI [13], [15], [89]. The conductivity further increased to $7.8 \times 10_{-6}$ and $1.15 \times 10-5 \mathrm{~S} \mathrm{~cm}-1$ after 4 and 5 minutes of plasma functionalization, respectively. The trend of exponential decrease in the sheet resistance and the corresponding increase in the conductivity of the Spiro-OMeTAD films post-plasma exposure is shown in Figure 11.

\section{Kelvin Probe Force Microscopy}

Kelvin Probe Force Microscopy (KPFM) is one of the most commonly used nanoscale electrical characterisation techniques for the quantitative determination of the work function of surfaces with respect to the conducting tip used for the measurement. This versatile technique has been extensively used to probe the variation in cross-sectional surface potential, charge separation, charge transport, map the local contact potential difference, and electronic structure at the interfaces of PSCs [100]-[103]. 
In this work KPFM was used to measure the work function variation of Spiro-OMeTAD films due to time-dependent plasma exposure. Initially the work function of the Pt/Ir coated tip was estimated by mapping the surface potential of freshly cleaved HOPG sample. The AMKPFM maps revealed that the work function of the Pt/Ir tip is $5.21 \mathrm{eV}$. The contact potential difference is defined as:

$\Delta V_{C P D}=-\frac{\phi_{\text {tip }}-\phi_{\text {sample }}}{e} \quad \ldots \ldots .2$

where $\phi_{\text {tip }}$ is the work function of the KPFM tip, $\phi_{\text {sample }}$ is the work function of the sample, $e$ is the elementary charge, and $\Delta V_{C P D}$ is the measured contact potential difference.

Using this relation, the work function measurements were performed before and after plasma treatment. From the surface potential (SP) values it was concluded that the work function of the Spiro-OMeTAD thin films increased after oxygen plasma treatment. This indicates that the oxygen plasma induced $p$-type functionalization of the Spiro-OMeTAD films. However, in practice, the surface potential is affected by several factors and thus, the measured surface potential difference from KPFM, though accurate, has multiple contributions which renders this technique pseudo-quantitative. The work function is measured with respect to the cantilever tip and is therefore not an absolute value.

Figure S3 (a), shows the surface potential map of the pristine Spiro-OMeTAD film and Figure S3 (b) shows the distribution of surface potential. The histogram plot of the surface potential has been fitted with a Gaussian distribution profile and the mean value of this distribution can be considered as the average potential difference between the KPFM tip and the Spiro-OMeTAD film. The same technique was used for the calculation of the surface potential values for all the plasma-treated Spiro-OMeTAD samples. KPFM measurements were performed on the same sample before and after plasma treatment and the variation in surface 
potential due to plasma exposure was recorded as shown in Table 4. A wide variation in the surface potential values was observed. Only 3 minutes of plasma treatment resulted in a surface potential of $511.0 \mathrm{mV}$ which is $\sim 17.0 \mathrm{mV}$ more than that of the untreated film $(494.0 \mathrm{mV})$. The variation in surface potential increased to $49.0 \mathrm{mV}$ after 4 minutes of plasma functionalization before finally reducing to $14.0 \mathrm{mV}$ for 5 minutes samples. Thus, it was concluded that the work function of the Spiro-OMeTAD samples has increased after plasma treatment, which signifies p-type doping of Spiro-OMeTAD with oxygen plasma, albeit there is certain variation in the values with different time of treatment.

Table 4 Variation of the surface potential of pristine and plasma-treated Spiro-OMeTAD as measured with Kelvin Probe Force Microscopy.

\begin{tabular}{|c|c|c|c|}
\hline $\begin{array}{c}\text { Time of plasma } \\
\text { exposure }\end{array}$ & $\begin{array}{c}\text { SP pre-treatment } \\
(\mathbf{V})\end{array}$ & $\begin{array}{c}\text { SP post-treatment } \\
(\mathbf{V})\end{array}$ & $\begin{array}{c}\text { Change in SP } \\
(\mathbf{m V})\end{array}$ \\
\hline 3 mins & 0.494 & 0.511 & +17.0 \\
\hline 4 mins & 0.469 & 0.518 & +49.0 \\
\hline 5 mins & 0.456 & 0.470 & +14.0 \\
\hline
\end{tabular}

\section{Mechanism of increase in conductivity due to plasma treatment}

The low conductivity and hole mobility of Spiro-OMeTAD stems from the $s p 3$ hybridized $\mathrm{N}$ atom with pyramidal structures which leads to large intermolecular distances. Thus, charges have to move longer paths and hence the conductivity is lowered [104]. According to Shi et al. the lower hole mobility can also be explained in terms of the two types of steric hindrances which exist in unit cells of Spiro-OMeTAD and affect the $\pi-\pi$ stacking of the molecules [105]. Close $\pi-\pi$ stacking upon crystallization is prevented by the steric hindrance between two molecules in a single crystal unit cell whereas the formation of continuous $\pi-\pi$ stacking is 
prohibited by the hindrance between the outer fragments of each cell. The second mechanism can be attributed to the twisted nature of the central Spiro carbon. This discontinuity inhibits delocalization of charge carriers and charge transport occurs primarily by hopping from one Spiro-OMeTAD molecule to another [105].

Abate et al. have proposed the mechanism of Spiro-OMeTAD oxidation with chemical dopants such as Li-TFSI in presence of oxygen as [15]:

Spiro-OMeTAD $+\mathrm{O}_{2} \rightarrow$ Spiro-OMeTAD. $+\mathrm{O}_{2}$.

Eventually Spiro-OMeTAD+. interacts with the TFSI- anion during conventional chemical $p$ type doping procedure with any metal TFSI such as Li-TFSI, Na-TFSI or H-TFSI.

In this work, enhancement in conductivity of Spiro-OMeTAD after plasma treatment has been proposed to occur primarily as a result of an increase in the number of charge carriers and the diffusion of oxidants from the plasma jet into the Spiro-OMeTAD films. The SpiroOMeTAD+. radical formed after oxidation of the molecule in presence of oxygen, has $\delta+$ charge on triarylamine nitrogen which acts as a suitable site for attack by the numerous charged species such as radicals, ions, electrons present in the oxygen plasma. These charged species attack the $\delta_{+}$nitrogen of the triarylamine nitrogen site, providing additional charge carriers to the SpiroOMeTAD molecule and hence, the conductivity is enhanced post plasma treatment (Figure 12a). Moreover, interaction of the plasma jet with Spiro-OMeTAD may result in a loss of methyl groups, thus leading to the formation of quinoid structures with quaternary nitrogen $+\mathrm{N}=\mathrm{C}$, which is predicted to contribute to the enhancement in positive charge centres in the molecule as shown in Figure 12(b).

This study reports functionalization of Spiro-OMeTAD with APPJ for the first time and the mechanism of the plasma-surface interaction is still not clearly understood. The reason for the 
increase in conductivity of Spiro-OMeTAD post-processing may also be attributed to an increase in $\pi-\pi$ stacking, possibly due to the formation of hydrogen bonding between $\mathrm{OH} / \mathrm{O}_{2}$ species in plasma with the active centres of Spiro-OMeTAD molecule. It is believed that the oxygen species in the discharge enhances the delocalization of charge carriers and hence, contributes to an increase in conductivity post plasma exposure. Since atmospheric pressure plasma functionalization of Spiro-OMeTAD has not been undertaken before, the mechanism for the interaction of the highly energetic species present in the plasma jet with the molecule remains to be elucidated. Possibly the oxygen plasma promotes $\pi-\pi$ or $\mathrm{CH}-\pi$ interactions in Spiro-OMeTAD because of the presence of a multitude of different radicals/species, leading to an increased conductivity, since it is well known that aromatic rings can form $\mathrm{CH}-\pi$ interactions with alcohols, amines, amides and other molecules. Further research is being undertaken to understand the underlying mechanism of the plasma interaction with the hole transport molecule.

(a)

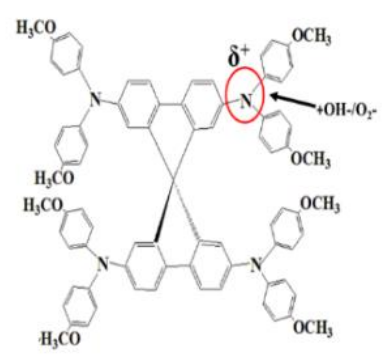

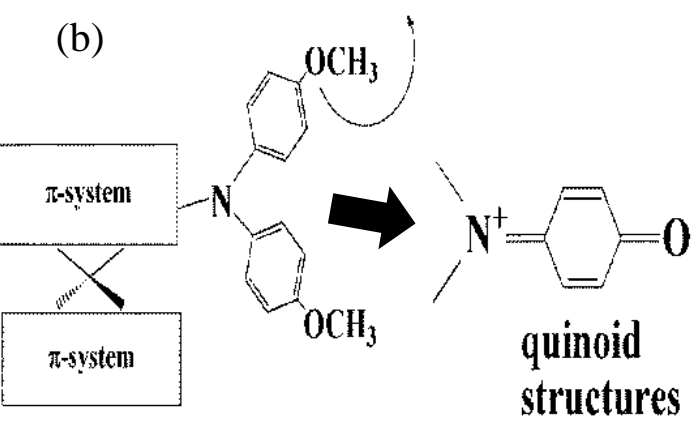

Figure 12 Proposed mechanism for the increase in conductivity due to plasma treatment (a) excess charge carrier centres from plasma jet and (b) formation of quinoid structures with quaternary $\mathrm{N}$ as positive charge centres after loss of methoxy groups from Spiro-OMeTAD during plasma-surface interaction.

\section{CONCLUSION}


A non-thermal atmospheric pressure plasma jet was used to functionalize and oxidise SpiroOMeTAD films. The hole conductivity is increased by more than one order of magnitude after 5 minutes of plasma treatment, reaching $10-5 \mathrm{~S} \mathrm{~cm}-1$. This conductivity value is comparable to that of $10-25 \%$ Li-TFSI doped Spiro-OMeTAD. Surface morphology suggests that the APPJ is a non-abrasive technique with variation in roughness between untreated and treated films being about $0.4 \mathrm{~nm}$. UV-Vis spectroscopy demonstrates the formation of Spiro-OMeTAD+ radical. The reduction in average phase value from EFM shows improvement in surface conductivity of plasma-functionalized samples. Oxidation of the Spiro-OMeTAD molecule and the variation in electronic properties is evident from the carbon, nitrogen and oxygen chemistry of the molecule probed with XPS. An increase in work function is observed in plasma-functionalized Spiro-OMeTAD films proving the $p$-type nature of the doping technique. These observations demonstrate the suitability of the atmospheric pressure plasma jet as a simple, scalable and effective means of doping and surface functionalization of Spiro-OMeTAD thin films. The enhancement in conductivity after treatment by positive ions and electrons in oxygen plasma, coupled with oxidation is expected to have positive implications on the hole transporting nature of the thin films without causing detrimental problems to the stability and resilience of the perovskite solar cells, in contrast to chemical dopants. However, further work is needed to elucidate the mechanism behind functionalization with atmospheric pressure plasma and to confirm electronic properties and the suitability of using plasma-treated Spiro-OMeTAD films as a hole transport layer in perovskite solar cells.

\section{Conflicts of interest}

There are no conflicts to declare.

\section{Acknowledgements}


Aruna Ivaturi would like to thank UK Research and Innovation (UKRI), Engineering and Physical Sciences Research Council (EPSRC) for the Fellowship grant (EP/P011500/1). SK acknowledges DST UKIERI, Royal Society and Royal Academy of Engineering for the funding support.

\section{References}

[1] National Renewable Energy Laboratory, "NREL Best Efficiency Chart.” 2020.

[2] F. Zhang et al., "Isomer-Pure Bis-PCBM-Assisted Crystal Engineering of Perovskite Solar Cells Showing Excellent Efficiency and Stability,” Adv. Mater., vol. 29, p. 1606806, 2017.

[3] F. Zhang et al., "Suppressing defects through the synergistic effect of a Lewis base and a Lewis acid for highly efficient and stable perovskite solar cells," Energy Environ. Sci., vol. 11, pp. 3480-3490, 2018.

[4] F. Zhang et al., "Self-Seeding Growth for Perovskite Solar Cells with Enhanced Stability," Joule, vol. 3, no. 6, pp. 1452-1463, 2019.

[5] N. H. Tiep, Z. Ku, and H. J. Fan, "Recent Advances in Improving the Stability of Perovskite Solar Cells," Adv. Energy Mater., vol. 6, no. 3, p. 1501420, Feb. 2016.

[6] Y.-K. Wang et al., "Dopant-Free Spiro-Triphenylamine/Fluorene as Hole-Transporting Material for Perovskite Solar Cells with Enhanced Efficiency and Stability," Adv. Funct. Mater., vol. 26, pp. 1375-1381, 2016.

[7] K. Liu et al., "Spiro[fluorene-9,9 0-xanthene]-based hole transporting materials for efficient perovskite solar cells with enhanced stability," Mater. Chem. Front., vol. 1, pp. 100-110, 2017.

[8] L. Liu et al., "Incorporation of rubidium cations into perovskite solar cells improves photovoltaic performance," Science (80-. )., vol. 354, no. 6309, p. 206, 2016.

[9] U. Bach et al., "Solid-state dye-sensitized mesoporous TiO2 solar cells with high photon-to-electron conversion efficiencies," Nature, vol. 395, no. 6702, pp. 583-585, 1998.

[10] K. Naito, "Quantitative Relations between Glass Transition Temperatures and Thermodynamic Parameters for Various Materials: Molecular Design for Nonpolymeric Organic Dye Glasses with Thermal Stability," Chem. Mater, vol. 6, pp. 2343-2350, 1994.

[11] U. Bach, K. De Cloedt, H. Spreitzer, and M. Grätzel, "Characterization of Hole Transport in a New Class of Spiro-Linked Oligotriphenylamine Compounds," $A d v$. Mater., vol. 12, no. 14, pp. 1060-1063, 2000.

[12] D. Poplavskyy and J. Nelson, "Nondispersive hole transport in amorphous films of methoxy-spirofluorene-arylamine organic compound," J. Appl. Phys., vol. 93, no. 1, 
pp. 341-346, 2003.

[13] H. J. Snaith and M. Grätzel, "Enhanced charge mobility in a molecular hole transporter via addition of redox inactive ionic dopant: Implication to dye-sensitized solar cells," Appl. Phys. Lett, vol. 89, p. 262114, 2006.

[14] J. Burschka et al., "Tris(2-(1H-pyrazol-1-yl)pyridine)cobalt(III) as p-type dopant for organic semiconductors and its application in highly efficient solid-state dye-sensitized solar cells.," J. Am. Chem. Soc., vol. 133, no. 45, pp. 18042-5, 2011.

[15] A. Abate et al., "Lithium salts as 'redox active' p-type dopants for organic semiconductors and their impact in solid-state dye-sensitized solar cells," Phys. Chem. Chem. Phys., vol. 15, pp. 2572-2579, 2013.

[16] L. K. Ono et al., "Air-Exposure-Induced Gas-Molecule Incorporation into SpiroMeOTAD Films," J. Phys. Chem. Lett., vol. 5, pp. 1374-1379, 2014.

[17] P. Docampo, S. Guldin, T. Leijtens, N. K. Noel, U. Steiner, and H. J. Snaith, "Lessons Learned: From Dye-Sensitized Solar Cells to All-Solid-State Hybrid Devices," Adv. Mater., vol. 26, no. 24, pp. 4013-4030, Jun. 2014.

[18] Z. Hawash, L. K. Ono, S. R. Raga, M. V. Lee, and Y. Qi, "Air-exposure induced dopant redistribution and energy level shifts in spin-coated Spiro-Meotad films," Chem. Mater., vol. 27, no. 2, pp. 562-569, 2015.

[19] A. Pellaroque et al., "Efficient and Stable Perovskite Solar Cells Using Molybdenum Tris(dithiolene)s as p-Dopants for Spiro-OMeTAD," ACS Energy Lett., vol. 2, no. 9, pp. 2044-2050, 2017.

[20] A. Abate, D. R. Staff, D. J. Hollman, H. J. Snaith, and A. B. Walker, "Influence of ionizing dopants on charge transport in organic semiconductors," Phys. Chem. Chem. Phys., vol. 16, pp. 1132-1138, 1132.

[21] B. Xu, J. Huang, H. A gren, L. Kloo, A. Hagfeldt, and L. Sun, "AgTFSI as p-type dopant for efficient and stable solid-state dye-sensitized and perovskite solar cells," ChemSusChem, vol. 7, no. 12, pp. 3252-3256, 2014.

[22] L.-H. Xie et al., "Unexpected One-Pot Method to Synthesize Spiro[fluorene-9,9'xanthene] Building Blocks for Blue-Light-Emitting Materials," Org. Lett., vol. 8, no. 13, pp. 2787-2790, 2006.

[23] R. Hock, T. Mayer, and W. Jaegermann, "P-type doping of spiro-MeOTAD with WO3 and the spiro-MeOTAD/WO3 interface investigated by synchrotron-induced photoelectron spectroscopy," J. Phys. Chem. C, vol. 116, no. 34, pp. 18146-18154, 2012.

[24] M. Li et al., "Copper Salts Doped Spiro-OMeTAD for High-Performance Perovskite Solar Cells," Adv. Energy Mater., vol. 6, no. 1601156, pp. 1-8, 2016.

[25] C. Chen et al., "Cu(II) Complexes as p-Type Dopants in Efficient Perovskite Solar Cells," ACS Energy Lett., vol. 2, pp. 497-503, 2017.

[26] W. H. Nguyen, C. D. Bailie, E. L. Unger, and M. D. McGehee, "Enhancing the hole- 
conductivity of spiro-OMeTAD without oxygen or lithium salts by using spiro(TFSI)2 in perovskite and dye-sensitized solar cells," J. Am. Chem. Soc., vol. 136, no. 31, pp. 10996-11001, 2014.

[27] L. Yang et al., "Cobalt Salt as Efficient Dopant for Spiro-MeOTAD in CesiumContaining Planar Perovskite Solar Cells," J. Nanosci. Nanotechnol., vol. 18, no. 4, pp. 2898-2902, 2018.

[28] J. Burschka, F. Kessler, M. K. Nazeeruddin, and M. Grä, "Co(III) Complexes as p-Dopants in Solid-State Dye-Sensitized Solar Cells," Chem. Mater., vol. 25, pp. 2986-2990, 2013.

[29] M. Xu, Y. Rong, Z. Ku, A. Mei, X. Li, and H. Han, "Improvement in Solid-State Dye Sensitized Solar Cells by p-Type Doping with Lewis Acid SnCl4," J. Phys. Chem. C, vol. 117, pp. 22492-22496, 2013.

[30] P. Wang et al., "Copper iodide as a potential low-cost dopant for spiro-MeOTAD in perovskite solar cells,” J. Mater. Chem. C, vol. 4, no. 4, pp. 9003-9008, 2016.

[31] X. Gu, Y. Li, Y. Mu, M. Zhang, T. Lu, and P. Wang, "FeCl3 as a low-cost and efficient p-type dopant of Spiro-OMeTAD for high performance perovskite solar cells," RSC Adv., vol. 8, pp. 9409-9413, 2018.

[32] R. Lindblad, "Electronic Structures and Energy Level Alignment in Mesoscopic Solar Cells : A Hard and Soft X-ray Photoelectron Spectroscopy Study,” 2014.

[33] B. Enright, C. Redmond, and D. Fitzmaurice, "Spectroscopic Determination of Flatband Potentials for Polycrystalline Ti02 Electrodes in Mixed Solvent Systems," J. Phys. Chem, vol. 98, pp. 6195-6200, 1994.

[34] S. N. Habisreutinger, T. Leijtens, G. E. Eperon, S. D. Stranks, R. J. Nicholas, and H. J. Snaith, "Enhanced Hole Extraction in Perovskite Solar Cells Through Carbon Nanotubes," J. Phys. Chem. Lett., vol. 5, pp. 4207-4212, 2014.

[35] S. N. Habisreutinger, T. Leijtens, G. E. Eperon, S. D. Stranks, R. J. Nicholas, and H. J. Snaith, "Carbon Nanotube/Polymer Composites as a Highly Stable Hole Collection Layer in Perovskite Solar Cells," Nano Lett., vol. 14, pp. 5561-5568, 2014.

[36] S. Wang et al., "Role of 4-tert-Butylpyridine as a Hole Transport Layer Morphological Controller in Perovskite Solar Cells," Nano lett, vol. 16, no. 9, pp. 5594-5600, 2016.

[37] S. N. Habisreutinger, N. K. Noel, H. J. Snaith, and R. J. Nicholas, "Investigating the role of 4-tert butylpyridine in perovskite solar cells," Adv. Energy Mater., vol. 7, no. 1, p. 7934, 2017.

[38] N. Hayashi, S. Tsutsui, T. Tomari, and W. Guan, "Sterilization of Medical Equipment Using Oxygen Radicals Produced by Water Vapor RF Plasma," IEEE Trans. Plasma Sci., vol. 36, no. 4, p. 1302, 2008.

[39] J. Romero-Mangado et al., "Efficacy of atmospheric pressure dielectric barrier discharge for inactivating airborne pathogens," J. Vac. Sci. Technol. A Vacuum, Surfaces, Film., vol. 35, no. 35, pp. 41101-41603, 2017. 
[40] I. Beams, P. S. Engineering, and H. Kong, "Guest Editorial Special Issue on PlasmaBased Surface Modification and Treatment Technologies," vol. 37, no. 7, pp. 1121$1122,2009$.

[41] A. Sarani, A. Y. Nikiforov, N. De Geyter, R. Morent, and C. Leys, "Surface modification of polypropylene with an atmospheric pressure plasma jet sustained in argon and an argon/water vapour mixture," Appl. Surf. Sci., vol. 257, no. 20, pp. 87378741, 2011.

[42] A. Sarani et al., "Surface modification of PTFE using an atmospheric pressure plasma jet in argon and argon+CO2," Surf. Coatings Technol., vol. 206, no. 8-9, pp. 2226$2232,2012$.

[43] Z. Machala et al., "Emission spectroscopy of atmospheric pressure plasmas for biomedical and environmental applications," J. Mol. Spectrosc., vol. 243, pp. 194-201, 2007.

[44] G. E. Morfill, M. G. Kong, and J. L. Zimmermann, "Focus on plasma medicine," New J. Phys., vol. 11, 2009.

[45] J. Laimer and H. Störi, "Recent advances in the research on non-equilibrium atmospheric pressure plasma jets," Plasma Process. Polym., vol. 4, no. 3, pp. 266-274, 2007.

[46] T. Review, "Nonthermal plasma synthesis of semiconductor nanocrystals," J. Phys. D Appl. Phys, vol. 42, p. 113001, 2009.

[47] D. Szabó and S. Schlabach, "Microwave Plasma Synthesis of Materials-From Physics and Chemistry to Nanoparticles: A Materials Scientist's Viewpoint," Inorganics, vol. 2, no. 3, pp. 468-507, 2014.

[48] X. Liang, Z. Fu, and S. Y. Chou, "Graphene Transistors Fabricated via TransferPrinting In Device Active-Areas on Large Wafer," Nano Lett., vol. 7, no. 12, pp. 38403844, 2007.

[49] J. Hwang et al., "Multilayered graphene anode for blue phosphorescent organic light emitting diodes," Appl. Phys. Lett, vol. 100, no. 133304, p. 1, 2012.

[50] H. Mao, R. Wang, J. Zhong, S. Zhong, and W. Chen, "Mildly O2 plasma treated CVD graphene as a promising platform for molecular sensing," Carbon N. Y., vol. 76, pp. 212-219, 2014.

[51] N. Rolston et al., "Improved stability and efficiency of perovskite solar cells with submicron flexible barrier films deposited in air," J. Mater. Chem. A, vol. 5, pp. 22975-22983, 2017.

[52] X. Xiao et al., "Argon Plasma Treatment to Tune Perovskite Surface Composition for High Efficiency Solar Cells and Fast Photodetectors," Adv. Mater., vol. 30, no. 9, pp. $1-7,2018$.

[53] F. Hilt, M. Q. Hovish, N. Rolston, K. Brü, C. J. Tassone, and R. H. Dauskardt, "Rapid route to efficient, scalable, and robust perovskite photovoltaics in air," Energy Environ. Sci., vol. 11, p. 2102, 2018. 
[54] S. Zhang et al., "Interface engineering of solution processed hybrid organohalide perovskite solar cells," ACS Appl. Mater. Interfaces, vol. 10, no. 25, pp. 21681-31687, 2018.

[55] Y. Nishihara, M. Chikamatsu, S. Kazaoui, T. Miyadera, and Y. Yoshida, "Influence of O2 plasma treatment on NiOx layer in perovskite solar cells," Jpn. J. Appl. Phys, vol. 57, pp. 4-7, 2018.

[56] H. M. Xiao-Mei Li, Kai-li Wang, Yu-Rong Jiang, Ying-Guo Yang, Xing-Yu Gao, "The furrowed hole-transport layer using Argon plasma in the inverted perovskite solar cell," New J. Chem., vol. 43, pp. 14625-14633, 2019.

[57] Y. Wang, H. Qu, C. Zhang, and Q. Chen, "Rapid Oxidation of the Hole Transport Layer in Perovskite Solar Cells by A Low-Temperature Plasma," Sci. Rep., vol. 9, no. 459, pp. 1-9, 2019.

[58] J.-H. B. Dong In Kim, Ji Won Lee, Rak Hyun Jeong, Sang-hun Nam, Ki-Hwan Hwang, "Oxygen-plasma processed Spiro-OMeTAD toward high performance for perovskite solar cell,” Surf. Coat. Technol., vol. 357, pp. 189-194, 2019.

[59] J. C. and D. W. Hao Qu, Gao Zhao, Yumeng Wang, Lijuan Liang, Long Zhang, Wenya Liu, Chunmei Zhang, Chen Niu, Yi Fang, Jiazi Shi, "Plasma-Exposure-Induced Mobility Enhancement of LiTFSI-Doped Spiro-OMeTAD Hole Transport Layer in Perovskite Solar Cells and Its Impact on Device Performance," Materials (Basel)., vol. 12, p. 3142, 2019.

[60] C. D. Bailie, E. L. Unger, S. M. Zakeeruddin, M. Grä Tzel, and M. D. Mcgehee, "Meltinfiltration of spiro-OMeTAD and thermal instability of solid-state dye-sensitized solar cells," Phys. Chem. Chem. Phys., vol. 4864, no. 16, pp. 4864-4870, 2014.

[61] E. J. Juárez-Pérez, M. R. Leyden, S. Wang, L. K. Ono, Z. Hawash, and Y. Qi, "Role of the Dopants on the Morphological and Transport Properties of Spiro-MeOTAD Hole Transport Layer," Chem. Mater., vol. 28, pp. 5702-5709, 2016.

[62] S. Yang, Y. Wang, P. Liu, Y.-B. Cheng, H. J. Zhao, and H. G. Yang, "Functionalization of perovskite thin films with moisture-tolerant molecules," Nat. Energy, vol. 1, p. 15016, Jan. 2016.

[63] V. Schulz-Von Der Gathen et al., "Spatially resolved diagnostics on a microscale atmospheric pressure plasma jet," J. Phys. D. Appl. Phys., vol. 41, no. 19, p. 194004, 2008.

[64] D. Marinov and N. S. J. Braithwaite, "Power coupling and electrical characterization of a radio-frequency micro atmospheric pressure plasma jet," Plasma Sources Sci.

Technol., vol. 23, no. 6, p. 062005, 2014.

[65] N. Krstulovi, I. Labazan, S. Miloševi, U. Cvelbar, A. Vesel, and M. Mozetič, "Optical emission spectroscopy characterization of oxygen plasma during treatment of a PET foil," J. Phys. D Appl. Phys, vol. 39, pp. 3799-3804, 2006.

[66] V. Milosavljevic, M. Donegan, P. Cullen, D. Dowling, and D. Diagnostics, "Diagnostics of an O2-He RF Atmospheric Plasma Discharge by Spectral Emission," J. Phys. Soc. Japan, vol. 83, no. 65940, pp. 1-8, 2014. 
[67] R. E. Walkup, K. L. Saenger, and G. S. Selwyn, "Studies of atomic oxygen in O2 +CF 4 rf discharges by two-photon laser-induced fluorescence and optical emission spectroscopy," J. Chem. Phys., vol. 84, no. 5, pp. 2668-2674, 1986.

[68] P. H. Lofthus, Alf; Krupenie, "The spectrum of molecular nitrogen," J. Phys. chem. Ref. Data, vol. 6, no. 1, pp. 113-307, 1977.

[69] M. Thiyagarajan, A. Sarani, and C. Nicula, "Optical emission spectroscopic diagnostics of a non-thermal atmospheric pressure helium-oxygen plasma jet for biomedical applications," J. Appl. Phys., vol. 113, no. 23, pp. 233302-1, 2013.

[70] J. and N. A. T. Kramida, A., Ralchenko, Yu., Reader, "NIST: Atomic Spectra Database - Version 5.5.2,” 2018. [Online]. Available: https://physics.nist.gov/asd. [Accessed: 05Mar-2018].

[71] M. K. Singh, A. Ogino, M. Nagatsu, X. Yang, X. Chang, and R. Tei, "Inactivation factors of spore-forming bacteria using low-pressure microwave plasmas in an N2 and O2 gas mixture," New J. Phys, vol. 11, no. 11, p. 115027, Nov. 2009.

[72] W. Gao, L. B. Alemany, L. Ci, and P. M. Ajayan, "New insights into the structure and reduction of graphite oxide.," Nat. Chem., vol. 1, no. 5, pp. 403-408, 2009.

[73] A. Bagri, C. Mattevi, M. Acik, Y. J. Chabal, M. Chhowalla, and V. B. Shenoy, "Structural evolution during the reduction of chemically derived graphene oxide," Nat. Chem., vol. 2, no. 7, pp. 581-587, 2010.

[74] J. Gardella, S. A. Ferguson, and R. L. Chin, "pi *-pi Shakeup Satellites for the Analysis of Structure and Bonding in Aromatic Polymers by X-Ray Photoelectron Spectroscopy," Appl. Spectrosc., vol. 40, no. 2, pp. 224-232, 1986.

[75] S. R. Kelemen, K. D. Rose, and P. J. Kwiatek, "Carbon aromaticity based on XPS II to II*signal intensity,” Appl. Surf. Sci., vol. 64, no. 2, pp. 167-174, 1993.

[76] E. M. J. Johansson, M. Odelius, P. G. Karlsson, H. Siegbahn, A. Sandell, and H. Rensmo, "Interface electronic states and molecular structure of a triarylamine based hole conductor on rutile TiO2(110)," J. Chem. Phys., vol. 128, no. 18, 2008.

[77] R. Schoelin, M. H. Karlsson, S. K. Eriksson, H. Siegbahn, E. M. J. Johansson, and H. Rensmo, "Energy level shifts in spiro-OMeTAD molecular thin films when adding LiTFSI," J. Phys. Chem. C, vol. 116, no. 50, pp. 26300-26305, 2012.

[78] G. Lota, B. Grzyb, H. Machnikowska, J. Machnikowski, and E. Frackowiak, "Effect of nitrogen in carbon electrode on the supercapacitor performance," Chem. Phys. Lett., vol. 404, pp. 53-58, 2005.

[79] D. Hulicova-Jurcakova, M. Kodama, S. Shiraishi, H. Hatori, Z. H. Zhu, and G. Q. Lu, "Nitrogen-enriched nonporous carbon electrodes with extraordinary supercapacitance," Adv. Funct. Mater., vol. 19, no. 11, pp. 1800-1809, 2009.

[80] X. Wang, J. S. Lee, Q. Zhu, J. Liu, Y. Wang, and S. Dai, “Ammonia-Treated Ordered Mesoporous Carbons as Catalytic Materials for Oxygen Reduction Reaction," Chem. Mater, vol. 22, pp. 2178-2180, 2010. 
[81] X. Wang et al., "Nitrogen-enriched ordered mesoporous carbons through direct pyrolysis in ammonia with enhanced capacitive performance," J. Mater. Chem. A, vol. 1, p. 7920, 2013.

[82] Z. Chen, D. Higgins, and Z. Chen, "Nitrogen doped carbon nanotubes and their impact on the oxygen reduction reaction in fuel cells," Carbon N. Y., vol. 48, pp. 3057-3065, 2010 .

[83] E. J. Biddinger and U. S. Ozkan, "Role of Graphitic Edge Plane Exposure in Carbon Nanostructures for Oxygen Reduction Reaction," J Phys Chem C, vol. 114, pp. 1530615314, 2010.

[84] L. Lai et al., "Exploration of the active center structure of nitrogen-doped graphenebased catalysts for oxygen reduction reaction," Energy Environ. Sci., vol. 5, pp. 79367942, 2012.

[85] M. Thelakkat, "Star-shaped, dendrimeric and polymeric triarylamines as photoconductors and hole transport materials for electro-optical applications," Macromol. Mater. Eng., vol. 287, no. 7, pp. 442-461, 2002.

[86] B. Cecilia Mattevi et al., "Evolution of Electrical, Chemical, and Structural Properties of Transparent and Conducting Chemically Derived Graphene Thin Films," Adv. Funct. Mater., vol. 19, pp. 2577-2583, 2009.

[87] A. Ganguly, S. Sharma, P. Papakonstantinou, and J. Hamilton, "Probing the Thermal Deoxygenation of Graphene Oxide Using High-Resolution In Situ X-ray-Based Spectroscopies," J. Phys. Chem. C, vol. 115, pp. 17009-17019, 2011.

[88] N. M. Barkoula, B. Alcock, N. O. Cabrera, and T. Peijs, "The Effect of Carboxylic Acid Group on Conductivity of the Aromatic Polyazomethines and Char Composites," Polym. Polym. Compos., vol. 16, no. 2, pp. 101-113, 2008.

[89] Z. Li et al., "Acid Additives Enhancing the Conductivity of Spiro-OMeTAD Toward High-Efficiency and Hysteresis-Less Planar Perovskite Solar Cells," Adv. Energy Mater., vol. 7, p. 1601451, Feb. 2016.

[90] S. Wang, W. Yuan, and Y. S. Meng, "Spectrum-Dependent Spiro-OMeTAD Oxidization Mechanism in Perovskite Solar Cells," ACS Appl. Mater. Interfaces, vol. 7, pp. 24791-24798, 2015.

[91] S. Fantacci, F. De Angelis, M. K. Nazeeruddin, and M. Grätzel, "Electronic and optical properties of the spiro- MeOTAD hole conductor in its neutral and oxidized forms : a DFT / TDDFT investigation," J. Phys. Chem. C, vol. 115, pp. 23126-23133, 2011.

[92] O. Kazakova, V. Panchal, and T. Burnett, "Epitaxial Graphene and Graphene-Based Devices Studied by Electrical Scanning Probe Microscopy," Crystals, vol. 3, pp. 191233, Mar. 2013.

[93] S. E. S. E. Yalcin et al., "Direct Imaging of Charge Transport in Progressively Reduced Graphene Oxide Using Electrostatic Force Microscopy," ACS Nano, vol. 9, no. 0, pp. 2981-8, 2015.

[94] H. Jeong, K. M. Lee, Y. H. Ahn, S. Lee, and J. Y. Park, "Non-Contact Local 
Conductance Mapping of Individual Graphene Oxide Sheets during the Reduction Process," J. Phys. Chem. Lett., vol. 6, no. 13, pp. 2629-2635, 2015.

[95] R. Vidyasagar, B. Camargo, E. Pelegova, K. Romanyuk, and A. L. Kholkin, "Controlling Surface Potential of Graphene Using dc Electric Field," KnE Mater. Sci., vol. 1, no. 1, p. 183, 2016.

[96] H. Jeong, Y. H. Ahn, S. Lee, and J.-Y. Park, "Quantitative non-contact voltage profiling of quasi one-dimensional nanoelectronic devices," Appl. Phys. Lett., vol. 104, pp. 213102-1, 2014.

[97] J. M. R. Weaver and D. W. Abraham, "High resolution atomic force microscopy potentiometry High resolution atomic force microscopy potentiometry," J. Vac. Sci. Technol. B, vol. 9, pp. 1559-2921, 1991.

[98] A. Kuwabara, S. Kuroda, and H. Kubota, "Polymer Surface Treatment by Atmospheric Pressure Low Temperature Surface Discharge Plasma: Its Characteristics and Comparison with Low Pressure Oxygen Plasma Treatment," Plasma Chem. Plasma Process., vol. 28, no. 2, pp. 263-271, 2008.

[99] S. Reuter, K. Niemi, V. Schulz-Von Der Gathen, and H. F. Döbele, "Generation of atomic oxygen in the effluent of an atmospheric pressure plasma jet," Plasma Sources Sci. Technol., vol. 18, pp. 1-20, 2009.

[100] P. Qin et al., "Yttrium-substituted nanocrystalline TiO2 photoanodes for perovskite based heterojunction solar cells," Nanoscale, vol. 6, no. 3, pp. 1508-1514, 2014.

[101] A. Dymshits, A. Henning, G. Segev, Y. Rosenwaks, and L. Etgar, "The electronic structure of metal oxide/ organo metal halide perovskite junctions in perovskite based solar cells," Sci. Rep., vol. 5, no. 8704, pp. 1-6, 2015.

[102] C.-S. Jiang et al., "Carrier separation and transport in perovskite solar cells studied by nanometre-scale profiling of electrical potential," Nat. Commun., vol. 6, no. 8397, pp. $1-10,2015$.

[103] P. Solar and C. With, "Revealing the Working Mechanisms of Planar Surface Potential Profiling," IEEE J. Photovoltaics, vol. 8, no. 1, pp. 125-131, 2018.

[104] Y. Liu et al., "A dopant-free organic hole transport material for efficient planar heterojunction perovskite solar cells," J. Mater. Chem. A, vol. 3, pp. 11940-11947, 2015.

[105] D. Shi et al., "Spiro-OMeTAD single crystals: Remarkably enhanced charge-carrier transport via mesoscale ordering," Sci. Adv., vol. 2, no. 4, pp. 1-6, 2016. 


\section{Efficient hole transport material formed by atmospheric pressure plasma functionalization of Spiro-OMeTAD}

Paheli Ghosh a, Aruna Ivaturi b*, Debabrata Bhattacharya c, James Bowen a, Tony Nixon a, Jan Kowal a Nicholas St. J. Braithwaite d and Satheesh Krishnamurthy a* a School of Engineering and Innovation, The Open University, Milton Keynes, MK7 6AA, United Kingdom

b Smart Materials Research and Device Technology (SMaRDT) Group, Department of Pure and Applied Chemistry, University of Strathclyde, Thomas Graham building, Glasgow, G1 1XL, United Kingdom

c School of Aerospace, Transport and Manufacturing, Cranfield University, MK43 0AL, United Kingdom

d School of Physical Sciences, The Open University, Milton Keynes, MK7 6AA, United Kingdom.

KEYWORDS: atmospheric pressure plasma jet, conductivity, functionalization, hole transport materials, perovskite solar cells, XPS, EFM, KPFM 


\section{Supplementary information}
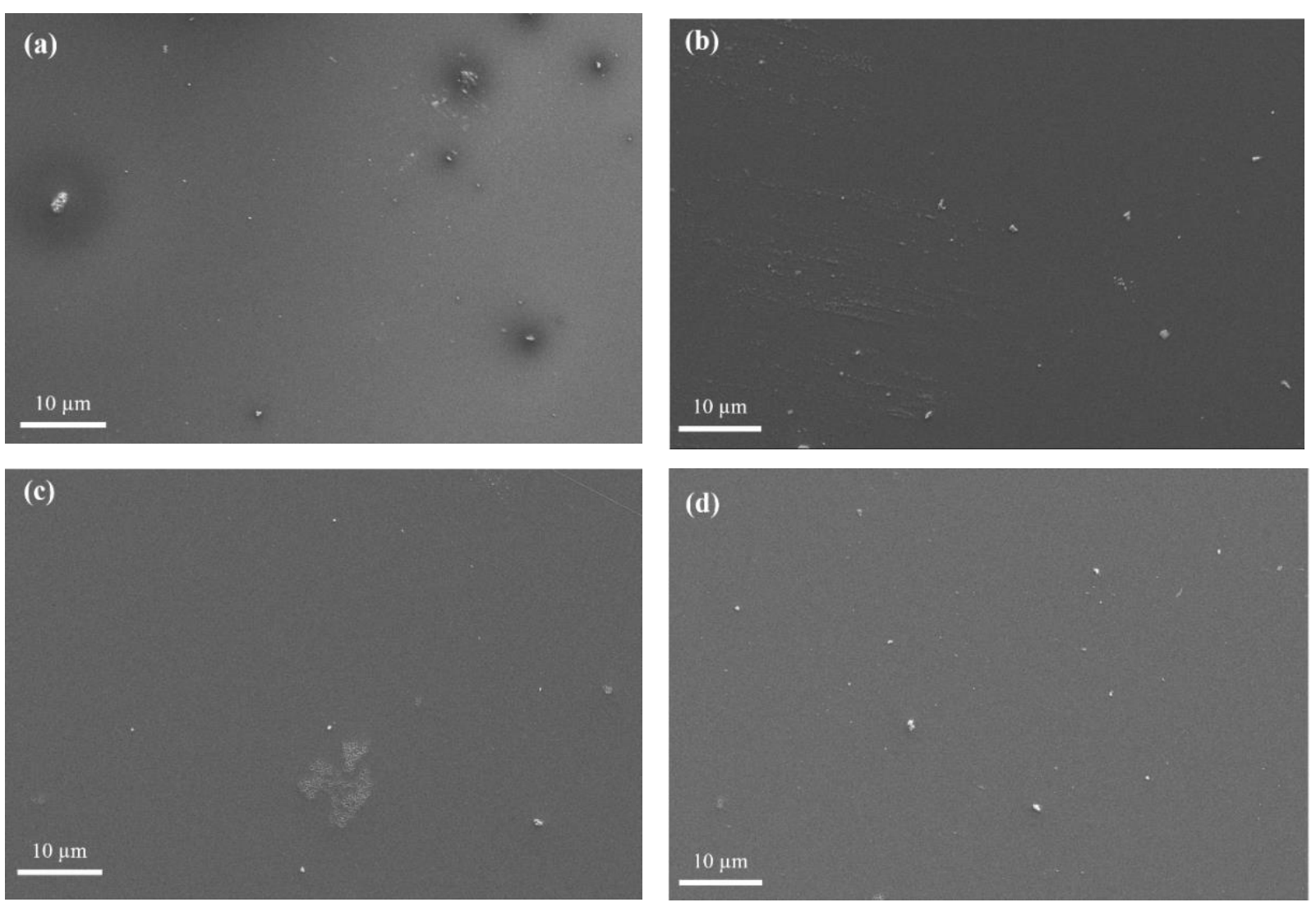

Figure S13 SEM images of untreated and plasma-treated spiro-OMeTAD (3, 4 and 5 mins - b, c and d, respectively).

There was no noticeable variation in the surface morphology before and after plasma treatment as observed using scanning electron microscopy (Figure S1). The films were very uniform, except for the presence of silicon dust on the surface (observed as small dots in the figures) and lacked the presence of pin hole defects usually reported in chemically doped spiro-OMeTAD thin films. These results demonstrate the non-abrasive nature of the atmospheric pressure plasma technique used in this manuscript. 

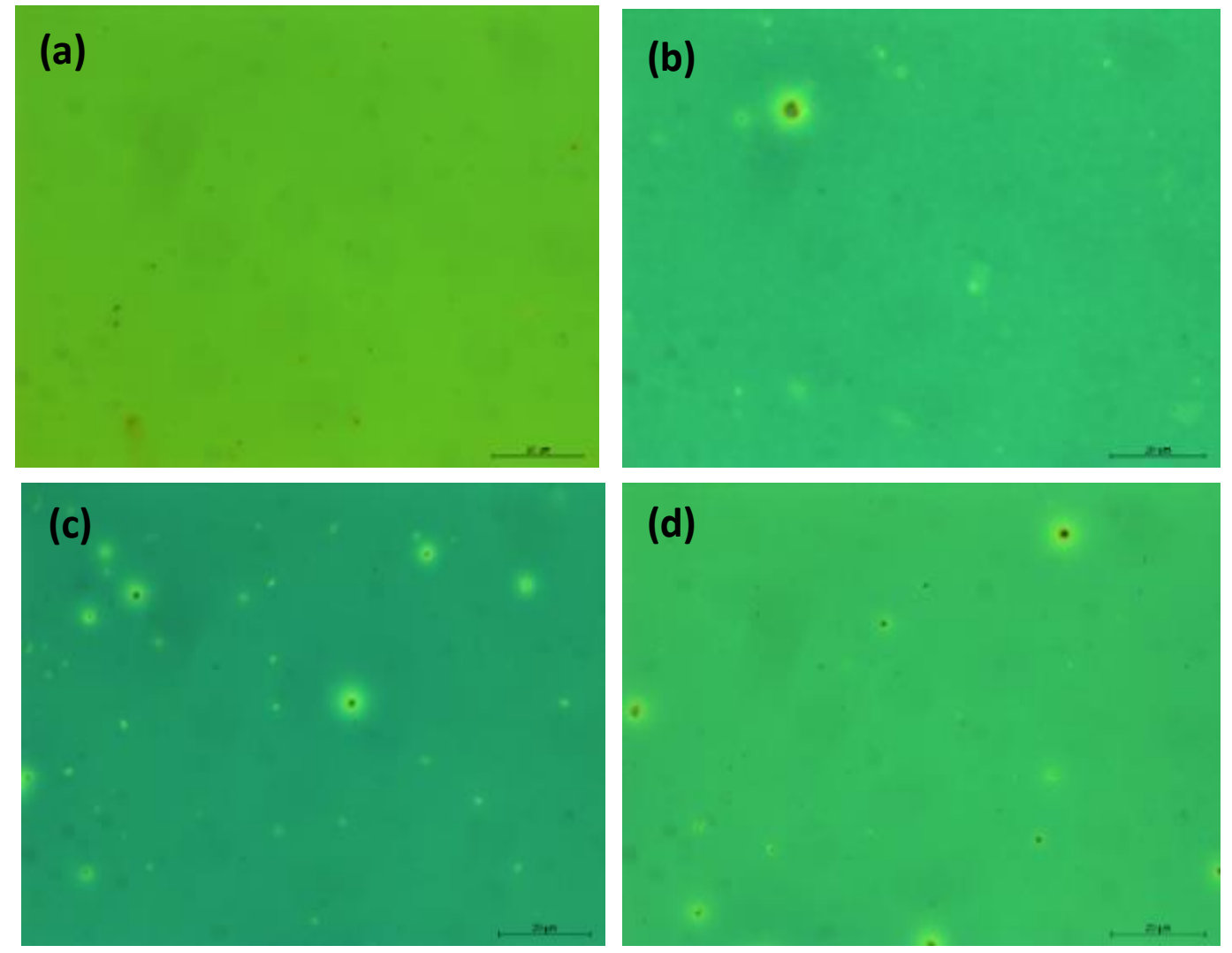

Figure S14 Optical images of untreated and plasma-treated spiro-OMeTAD (3, 4, 5 minutes of treatment time - b, c and d, respectively).

The change in colour from the as-deposited film to the plasma-functionalized film was observed with optical microscopy (Figure S2). The variation in colour of the films post-treatment from greenish to bluish becomes appreciable as the time of plasma exposure increases to 4 and 5 minutes but there was no presence of pin holes on the surface. 

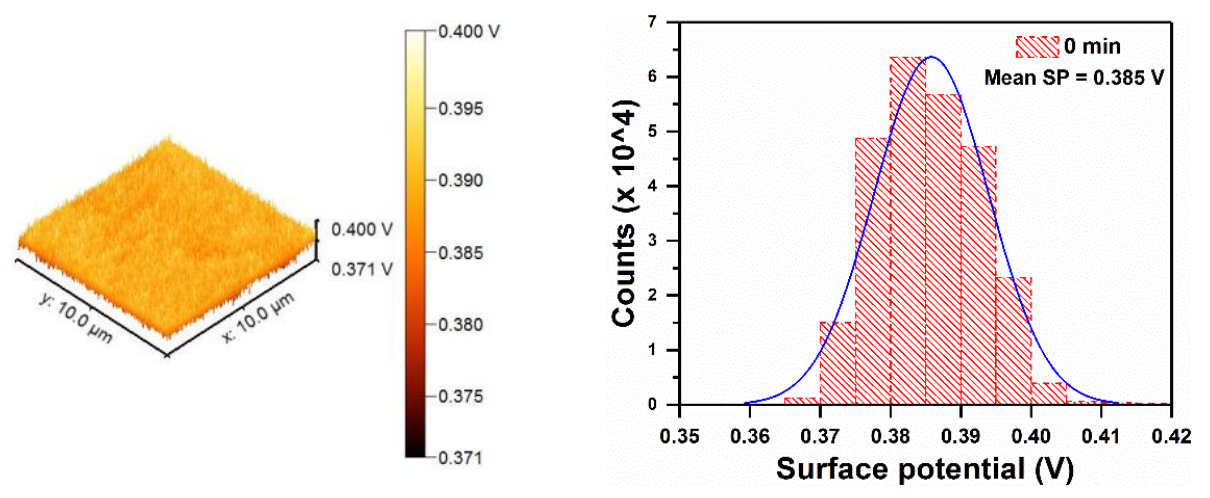

Figure S3 Representative image for the method of calculation of surface potential from KPFM data of pristine spiro-OMeTAD. 